\title{
Two-loop electroweak corrections to the Top-Quark Contribution to $\epsilon_{\mathrm{K}}$
}

\author{
Joachim Brod, Sandra Kvedaraitè and Zachary Polonsky \\ Department of Physics, University of Cincinnati, \\ 345 Clifton Ct, Cincinnati, OH 45221, U.S.A. \\ E-mail: joachim.brod@uc.edu, kvedarsa@ucmail.uc.edu, \\ polonsza@mail.uc.edu
}

ABSTRACT: The parameter $\epsilon_{K}$ measures $C P$ violation in the neutral kaon system. It is a sensitive probe of new physics and plays a prominent role in the global fit of the CabibboKobabyashi-Maskawa matrix. The perturbative theory uncertainty is currently dominated by the top-quark contribution. Here, we present the calculation of the full two-loop electroweak corrections to the top-quark contribution to $\epsilon_{K}$, including the resummation of QED-QCD logarithms. We discuss different renormalization prescriptions for the electroweak input parameters. In the traditional normalization of the weak Hamiltonian with two powers of the Fermi constant $G_{\mathrm{F}}$, the top-quark contribution is shifted by $-1 \%$.

Keywords: CP violation, Kaon Physics

ArXiv EPRINT: 2108.00017 


\section{Contents}

1 Introduction 1

2 Calculation of the two-loop electroweak matching corrections 4

2.1 Matching calculation 4

$\begin{array}{lll}2.2 & \text { Normalization to muon decay } & 7\end{array}$

3 Discussion of electroweak renormalization schemes $\quad 8$

3.1 MS scheme 8

$\begin{array}{llr}3.2 & \text { On-shell scheme } & 10\end{array}$

$\begin{array}{lll}3.3 & \text { Hybrid scheme } & 11\end{array}$

4 Inclusion of QCD $\quad 12$

$\begin{array}{lll}5 & \text { Conclusions } & 15\end{array}$

A Mass and field renormalization constants $\quad 16$

$\begin{array}{ll}\text { B Renormalization constants in the effective theory } & 17\end{array}$

$\begin{array}{ll}\text { C Scheme independence at order } \alpha & 19\end{array}$

$\begin{array}{ll}\text { D The full two-loop result } & 20\end{array}$

\section{Introduction}

The parameter $\epsilon_{K}$ describes CP violation in the neutral kaon system and is one of the most sensitive probes of new physics. It can be defined as [1]

$$
\epsilon_{K} \equiv e^{i \phi_{\epsilon}} \sin \phi_{\epsilon} \frac{1}{2} \arg \left(\frac{-M_{12}}{\Gamma_{12}}\right)
$$

Here, $\phi_{\epsilon}=\arctan \left(2 \Delta M_{K} / \Delta \Gamma_{K}\right)$ where $\Delta M_{K}$ and $\Delta \Gamma_{K}$ are the mass and lifetime differences of the weak eigenstates $K_{L}$ and $K_{S} . M_{12}$ and $\Gamma_{12}$ are the Hermitian and antiHermitian parts of the Hamiltonian that determines the time evolution of the neutral kaon system. The so-called short-distance contributions to $\epsilon_{K}$ enter the matrix element $M_{12}=-\left\langle K^{0}\left|\mathcal{L}_{f=3}^{\Delta S=2}\right| \bar{K}^{0}\right\rangle /\left(2 \Delta M_{K}\right)$, up to higher powers in the operator-product expansion.

Experimentally, $\epsilon_{K}$ is well-known, with absolute value $\left|\epsilon_{K}\right|=(2.228 \pm 0.011) \times 10^{-3}$ and an uncertainty at the permil level [2]. The standard model (SM) contributions to 
neutral kaon mixing are conveniently described by the effective $|\Delta S|=2$ Lagrangian with three active quark flavors,

$$
\mathcal{L}_{f=3}^{|\Delta S|=2}=-\frac{G_{\mathrm{F}}^{2} M_{W}^{2}}{4 \pi^{2}}\left[\lambda_{u}^{2} C_{S 2}^{\prime \prime u u}(\mu)+\lambda_{t}^{2} C_{S 2}^{\prime \prime t t}(\mu)+\lambda_{u} \lambda_{t} C_{S 2}^{\prime \prime u t}(\mu)\right] Q_{S 2}^{\prime \prime}+\text { h.c. }+\ldots,
$$

valid at scales around $\mu=2 \mathrm{GeV}$. Here,

$$
Q_{S 2}^{\prime \prime}=\left(\bar{s}_{L}^{\alpha} \gamma_{\mu} d_{L}^{\alpha}\right) \otimes\left(\bar{s}_{L}^{\beta} \gamma^{\mu} d_{L}^{\beta}\right)
$$

is the local $|\Delta S|=2$ operator, where $\alpha$ and $\beta$ are color indices, and the ellipsis denotes contributions of higher dimension local operators and non-local contributions of $|\Delta S|=1$ operators. The reason for the appearance of the double primes will become clear later. The elements of the Cabibbo-Kobayashi-Maskawa (CKM) matrix are combined into the parameters $\lambda_{i} \equiv V_{i s}^{*} V_{i d}$. The long-distance SM contributions are comprised by the hadronic matrix element of the local $|\Delta S|=2$ operator, and are given in terms of the kaon bag parameter $B_{K}=0.7625(97)$ [3]. Long-distance contributions that are not included in $B_{K}$ are parameterized by the correction factor $\kappa_{\epsilon}=0.94(2)$ [4].

The short-distance contributions are contained in the Wilson coefficients $C_{S 2}^{\prime \prime i j}$. In the $\mathrm{SM}$, the leading-order (LO) contributions to the Wilson coefficients are given by one-loop box diagrams [5]. Higher-order QCD corrections have been calculated in renormalizationgroup (RG) improved perturbation theory, using a slightly different representation of the $|\Delta S|=2$ effective Lagrangian [6], in terms of $\lambda_{c}$ and $\lambda_{t}$. However, it was shown recently [7] that the parameterization (1.2) in terms of $\lambda_{u}$ and $\lambda_{t}$ leads to a perturbative QCD uncertainty smaller by an order of magnitude. We will adopt this parameterization in this paper.

QCD corrections to the Wilson coefficients are known at next-to-leading order (NLO) for $C_{S 2}^{\prime \prime \prime t}[8]$ with a remaining uncertainty at the percent level, and at next-to-next-to-leading order (NNLO) for $C_{S 2}^{\prime \prime \prime}$ with a remaining uncertainty below one percent $[7,9,10]$. The coefficient $C_{S 2}^{\prime \prime \prime u}$ is also known at NNLO in QCD [7, 11], but has no effect on $\epsilon_{K}$ (it does contribute to the neutral kaon mass difference).

In anticipation of the NNLO QCD calculation of $C_{S 2}^{\prime \prime t t}[12]$ it is worthwhile to consider also the electroweak corrections to the effective Lagrangian (1.2). Without an explicit calculation, the electroweak renormalization scheme of the input parameters is left unspecified and amounts to an uncertainty of the order of a few percent — which can no longer be neglected at the current level of precision.

The electroweak corrections to $C_{S 2}^{\prime \prime \prime t}$ could, in principle, be adapted from the calculation of the corresponding corrections for $B$-meson mixing presented in ref. [13]. However, in our opinion, a reconsideration of the old calculation is worthwhile for a number of reasons. First, the application to $\epsilon_{K}$ involves a lower energy scale than the one relevant in $B$-meson mixing; accordingly, the QED-QCD resummation of the appearing logarithms might be necessary. Second, we will discuss the scheme dependence of the corrections in detail, since the usual proofs of scheme independence [6] fail in our case; this topic also has not been addressed before. Last, our calculation presents the first independent check of the results in ref. [13]. 
In this work, we calculate the coefficient $C_{S 2}^{\prime \prime t t}$, proportional to $\lambda_{t}^{2}$, to NLO in the electroweak interactions. This fixes the renormalization scheme of the electroweak input parameters contained in the prefactor $G_{\mathrm{F}}^{2} M_{W}^{2}$. In fact, when only considering QCD effects, there are several equivalent ways of rewriting the prefactor, using the tree-level relation

$$
G_{\mathrm{F}}=\frac{\pi \alpha}{\sqrt{2} M_{W}^{2} s_{w}^{2}}
$$

where $\alpha=e^{2} /(4 \pi)$ the electromagnetic coupling ${ }^{1}$ and $s_{w}^{2}=\sin ^{2} \theta_{w}$ with the weak mixing angle $\theta_{w}$. Essentially, this choice specifies which experimental data are used as parametric input for our prediction. The numerical difference between the different schemes is expected to be large at LO, as exemplified by the $5 \%$ difference between the on-shell and $\overline{\mathrm{MS}}$ values of $s_{w}^{2}$.

For our analysis, it is useful to write the effective Lagrangian in the following form:

$$
\mathcal{L}_{f=3}^{|\Delta S|=2}=\lambda_{t}^{2} c_{S 2}^{t t}(\mu) Q_{S 2}^{(\prime, \prime \prime)}+\text { h.c. }+\ldots,
$$

where the ellipsis now also includes contributions not proportional to $\lambda_{t}^{2}$. Using the treelevel relation (1.4), we express $c_{S 2}^{t t}$ in three different ways:

$$
c_{S 2}^{t t}(\mu)=-\frac{2 \pi^{2}}{M_{W}^{2} s_{w}^{4}} C_{S 2}^{t t}(\mu), \quad c_{S 2}^{t t}(\mu)=-\frac{G_{\mathrm{F}}}{\sqrt{2} s_{w}^{2}} C_{S 2}^{\prime t t}, \quad c_{S 2}^{t t}(\mu)=-\frac{G_{\mathrm{F}}^{2} M_{W}^{2}}{4 \pi^{2}} C_{S 2}^{\prime \prime t t} .
$$

As explained in section 2.2, this effectively absorbs different parts of the radiative corrections into the measured value of the muon decay rate. In the first and second relation in eq. (1.6) we have factored out the powers $(\alpha /(4 \pi))^{2}$ and $\alpha /(4 \pi)$, respectively, and absorbed them into rescaled operators, defined as

$$
Q_{S 2}=\left(\frac{\alpha}{4 \pi}\right)^{2}\left(\bar{s}_{L}^{\alpha} \gamma_{\mu} d_{L}^{\alpha}\right) \otimes\left(\bar{s}_{L}^{\beta} \gamma^{\mu} d_{L}^{\beta}\right)
$$

and

$$
Q_{S 2}^{\prime}=\frac{\alpha}{4 \pi}\left(\bar{s}_{L}^{\alpha} \gamma_{\mu} d_{L}^{\alpha}\right) \otimes\left(\bar{s}_{L}^{\beta} \gamma^{\mu} d_{L}^{\beta}\right),
$$

while $Q_{S 2}^{\prime \prime}$ has been defined in eq. (1.3). With these conventions, the RG evolution is described by the same anomalous dimension in all three cases, see section 4 , and the LO values

$$
C_{S 2}^{t t}(\mu)=C_{S 2}^{\prime t t}(\mu)=C_{S 2}^{\prime \prime t t}(\mu)=S\left(x_{t}\right)
$$

coincide. They are given by the modified [7] Inami-Lim box function [5]

$$
\mathscr{S}\left(x_{t}\right)=\frac{4 x_{t}-11 x_{t}^{2}+x_{t}^{3}}{4\left(x_{t}-1\right)^{2}}+\frac{3 x_{t}^{3}}{2\left(x_{t}-1\right)^{3}} \log x_{t},
$$

where $x_{t} \equiv m_{t}^{2} / M_{W}^{2}$, and we neglect a tiny correction of $\mathcal{O}\left(m_{c}^{2} / M_{W}^{2}\right) \sim 10^{-4}$ [7]. We will refer to the first normalization in eq. (1.6) as "A2", the second as "GF", and the third as

\footnotetext{
${ }^{1}$ Throughout this paper, the electromagnetic coupling is understood to be fixed in the five-flavor scheme at scale $\mu=M_{Z}$, unless otherwise noted.
} 
"GF2". While, at LO in the weak interactions, the three parameterizations are equivalent, the numerical prediction depends on the chosen normalization and the scheme of the input parameters. These arbitrary dependences will be mitigated to a large degree by the NLO electroweak corrections. The aim of this paper is to show this explicitly, and to provide an updated numerical prediction for $\epsilon_{K}$ including the electroweak corrections.

This paper is organized as follows. In section 2 we define the effective Lagrangian and provide details on our two-loop calculation. Section 3 contains the discussion of the various electroweak renormalization schemes and the error estimate on the electroweak corrections. In section 4 we include the effects of the strong interaction and discuss the scheme dependence of our result. We conclude in section 5. A number of appendices contains more details of our work. In appendix A we collect the explicit counterterms that were needed in the renormalization of the SM amplitude. The necessary counterterms in the effective theory are collected in appendix B. We give a formal proof of the scheme independence of the top-quark contribution to the $|\Delta S|=2$ amplitude including QED effects in appendix C. Finally, the full analytic two-loop result is presented in appendix D.

\section{Calculation of the two-loop electroweak matching corrections}

Our strategy is to perform the matching calculation using the "A2" normalization in the $\overline{\mathrm{MS}}$ scheme. We can then easily change to different normalization conventions and renormalization schemes for the input parameters; this will be discussed in detail in this section. The variation of the NLO results between the different prescriptions is used as one way to estimate the remaining theory uncertainty.

\subsection{Matching calculation}

We write the physical five-flavor effective Lagrangian, obtained by integrating out the top quark together with the weak gauge bosons $W$ and $Z$ as well as the Higgs boson, in the form ${ }^{2}$

$$
\mathcal{L}_{f=5}^{|\Delta S|=2}=-\frac{2 \pi^{2}}{M_{W}^{2} s_{w}^{4}} \lambda_{t}^{2} C_{S 2}^{t t}(\mu) Q_{S 2}+\text { h.c. }+\ldots
$$

The single physical operator contributing to our results has been defined in eq. (1.7). Our definition of the evanescent operators is given in appendix $\mathrm{B}$, thus fixing the renormalization scheme of our results.

The initial condition for the Wilson coefficient $C_{S 2}^{t t}$ at the electroweak scale is calculated from the difference of the $|\Delta S|=2$ amplitudes in the full SM and the five-flavor EFT. We expand the Wilson coefficients in the five-flavor EFT as

$$
C_{S 2}^{t t}=C_{S 2}^{t t,(0)}+\frac{\alpha^{(5)}}{4 \pi} C_{S 2}^{t t,(s e)}+\ldots
$$

where the superscript "(5)" emphasizes that the electroweak coupling is defined in the five-flavor scheme. We expand the amplitude in the full SM as

$$
\mathcal{A}^{t t}=-\frac{2 \pi^{2}}{M_{W}^{2} s_{w}^{4}} \lambda_{t}^{2} \sum_{i}\left(\mathcal{A}_{i}^{t t,(0)}+\frac{\alpha^{(6)}}{4 \pi} \mathcal{A}_{i}^{t t,(s e)}+\ldots\right)\left\langle Q_{i}\right\rangle^{(0)},
$$

\footnotetext{
${ }^{2}$ The RG evolution to the three-flavor effective Lagrangian will be considered in section 4 .
} 

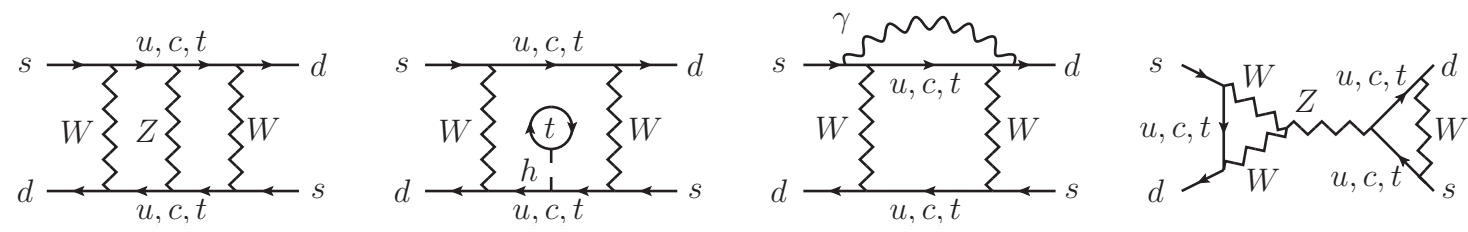

Figure 1. Sample two-loop Feynman diagrams contributing to the initial condition of $C_{S 2}^{\prime \prime t t}$.

in terms of the six-flavor coupling. This implies in the matching we have to include the finite threshold correction [14]

$$
\alpha^{(6)}=\alpha^{(5)}\left[1-\frac{\alpha^{(5)}}{4 \pi}\left(\frac{2}{3}+14 \log \frac{\mu}{M_{W}}-\frac{32}{9} \log \frac{\mu}{m_{t}}\right)\right],
$$

in order to express both results in terms of $\alpha^{(5)}$. The sum in eq. (2.3) runs over all operators in the basis.

The LO matching conditions are simply $\mathcal{A}_{i}^{t t,(0)}=C_{i}^{t t,(0)}$, and we find the following non-zero contributions (the terms of order $\epsilon$ in the physical Wilson coefficient are relevant for the two-loop calculation)

$$
\begin{aligned}
C_{S 2}^{t t,(0)}= & \frac{4 x_{t}-11 x_{t}^{2}+x_{t}^{3}}{4\left(x_{t}-1\right)^{2}}+\frac{3 x_{t}^{3}}{2\left(x_{t}-1\right)^{3}} \log x_{t} \\
& +\epsilon\left[\frac{4 x_{t}-11 x_{t}^{2}+x_{t}^{3}}{4\left(x_{t}-1\right)^{2}}+\frac{3 x_{t}^{3}}{2\left(x_{t}-1\right)^{3}} \log x_{t}\right] \log \left(\frac{\mu^{2}}{M_{W}^{2}}\right) \\
& +\epsilon\left[\frac{3 x_{t}^{3}-41 x_{t}^{2}-12 x_{t}}{8\left(x_{t}-1\right)^{2}}-\frac{x_{t}^{4}-14 x_{t}^{3}-8 x_{t}-4 x_{t}}{4\left(x_{t}-1\right)^{3}} \log x_{t}-\frac{3 x_{t}^{3}}{4\left(x_{t}-1\right)^{3}} \log ^{2} x_{t}\right] \\
& -4 \epsilon C_{E_{S 2}^{t t,(0)}} \\
C_{E_{S 2} t,(0)}^{t(0)} & -\frac{x_{t}+x_{t}^{2}}{4\left(x_{t}-1\right)^{2}}+\frac{x_{t}^{2}}{2\left(x_{t}-1\right)^{3}} \log x_{t} .
\end{aligned}
$$

We obtained this result by equating the amplitudes in the full SM and the five-flavor EFT defined above. We set all external momenta and fermion masses apart from the top quark to zero and employ dimensional regularization in $d=4-2 \epsilon$ dimensions for both ultraviolet (UV) and infrared (IR) divergences.

In the remainder of this section, we discuss the matching at NLO in the electroweak interactions. We split the NLO SM amplitude into several contributions as follows:

$$
\mathcal{A}_{Q_{S 2}^{\prime \prime}}^{t t,(s e)}=\mathcal{A}_{Q_{S 2}^{\prime \prime}}^{t t,(s e), \text { two-loop }}+\mathcal{A}_{Q_{S 2}^{\prime \prime}}^{t t,(s e), \text { countt }}+\mathcal{A}_{Q_{S 2}^{\prime \prime}}^{t t,(s e), \text { one-loop Z }}+\mathcal{A}_{Q_{S 2}^{\prime \prime}}^{t t,(s e), \text { tree-level Z }} .
$$

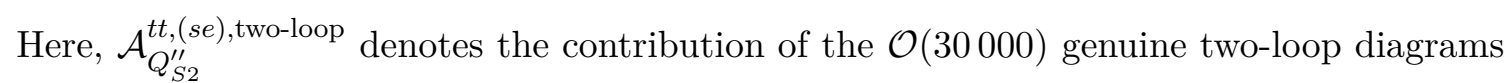
(see figure 1 for sample Feynman diagrams). The counterterm contributions arising from 

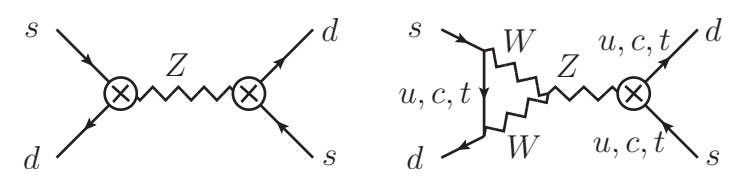

Figure 2. Sample Feynman diagrams contributing to the off-diagonal field renormalization: $\mathcal{A}_{Q_{S 2}^{t t}}^{\prime \prime}\left(\right.$ se), tree-level Z (left panel), and $\mathcal{A}_{Q_{S 2}^{\prime \prime}}^{t t,(s e) \text {,one-loop Z }}$ (right panel). The circled cross denotes a counterterm insertion.

the renormalization of the parameters in the LO result are

$$
\mathcal{A}_{Q_{S 2}^{\prime \prime}}^{t t,(s e), \text { countt }}=\left[\left(4 \frac{\delta e}{e}-2 \frac{\delta s_{w}^{2}}{s_{w}^{2}}-\frac{\delta M_{W}^{2}}{M_{W}^{2}}\right) \mathcal{A}_{Q_{S 2}^{\prime \prime}}^{t t,(0)}+\delta m_{t} \frac{\partial \mathcal{A}_{Q_{S 2}^{\prime \prime}}^{t t,(0)}}{\partial m_{t}}+\delta M_{W}^{2} \frac{\partial \mathcal{A}_{Q_{S 2}^{\prime \prime}}^{t t,(0)}}{\partial M_{W}^{2}}\right]
$$

The explicit forms of the renormalization constants are given in appendix A. We have included all tadpole graphs in both the full two-loop amplitude and the counterterms, such that the renormalization constants are gauge independent. The contributions of external field renormalization are comprised by $\mathcal{A}_{Q_{S 2}^{\prime \prime}}^{t t,(s e) \text {,tree-level Z }}$ and $\mathcal{A}_{Q_{S 2}^{\prime \prime}}^{t t, \text { (se),one-loop Z }}$ (see figure 2 for sample diagrams, and appendix A for the explicit form of the field renormalization constants). We include finite matching contributions in the renormalization of the external fields, such that the fields are normalized in the same way in the full and effective theories (cf. ref. [15]).

As an additional check, we calculated all one-loop diagrams with single counterterm insertions, including those corresponding to unphysical Goldstone boson field and mass renormalizations as well as Goldstone- $W$ mixing. After a careful application of the Slavnov-Taylor Identities [13], we analytically verified that the two methods of calculating $\mathcal{A}_{Q_{S 2}^{\prime \prime}}^{t t,(s e), \text { countt }}$ are identical.

Parameterizing the matrix elements in the EFT as

$$
\left\langle Q_{i}\right\rangle=\sum_{j} r_{i j}\left\langle Q_{j}\right\rangle^{(0)}
$$

where $\left\langle Q_{j}\right\rangle^{(0)}$ denotes the tree-level matrix element, and writing

$$
r_{i j}=\delta_{i j}+\frac{\alpha^{(5)}}{4 \pi} r_{i j}^{(s e)}+\ldots
$$

the NLO matching condition is

$$
C_{i}^{t t,(s e)}=\mathcal{A}_{i}^{t t,(s e)}-\sum_{j} C_{j}^{t t,(0)}\left(r_{j i}^{(s e)}+Z_{j i}^{(s e)}\right),
$$

where $Z_{j i}^{(s e)}$ denotes the renormalization constants for the Wilson coefficients; their explicit form is given in appendix B. The final result for $C_{i}^{t t,(s e)}$ is given in appendix D.

All diagrams have been calculated using self-written FORM [16] routines, implementing the two-loop recursion presented in refs. [17, 18]. The amplitudes were generated using qgraf [19]. As a check on our calculation, we verified that both IR and UV divergences 
cancel in the matching, yielding a finite result. We checked analytically that the full amplitude is independent of the matching scale to the considered order in the electroweak interaction.

\subsection{Normalization to muon decay}

When considering the prediction of low-energy observables at higher orders in the electroweak interactions, it is often advantageous to employ the muon lifetime as one of the experiments fixing the values of the input parameters. By a suitable normalization of the results, this allows for the absorption of parts of the radiative corrections into the muon lifetime measurement [20].

In the context of our calculation, we regard the Fermi constant $G_{\mathrm{F}}$ as the Wilson coefficient of muon decay in the Fermi effective theory [21]. We write the effective Lagrangian as

$$
\mathcal{L}_{\mathrm{F}}=-\frac{4}{\sqrt{2}} \frac{\pi \alpha}{\sqrt{2} M_{W}^{2} s_{w}^{2}} G_{\mu} Q_{\mu}+\text { h.c. },
$$

where

$$
Q_{\mu}=\left(\bar{\nu}_{\mu, L} \gamma^{\mu} \mu_{L}\right)\left(\bar{\nu}_{e, L} \gamma_{\mu} e_{L}\right)
$$

and we expand

$$
G_{\mu}=G_{\mu}^{(0)}+\frac{\alpha}{4 \pi} G_{\mu}^{(s e)}+\ldots
$$

The LO matching then just yields $G_{\mu}^{(0)}=1$, while a one-loop matching calculation gives

$$
\begin{aligned}
G_{\mu}^{(s e)}= & \frac{144 x^{2} z^{2}-18 x y z^{2}-21 y^{2} z^{2}+15 y z^{2}+14 y z+16 y-12 z^{2}-24}{24 y(z-1)} \\
& -\frac{22 y^{2} z^{3}-53 y^{2} z^{2}+28 y^{2} z-7 y z^{2}+38 y z-28 y-12 z+12}{4 y(z-1)^{2}(y z-1)} \log (z) \\
& +\frac{-108 x^{2} z^{2}+27 x y z^{2}-32 y z+32 y}{18 y(z-1)} \log (x)+\frac{3 y^{2} z^{3}-6 y z^{2}}{4(z-1)(y z-1)} \log (y) \\
& +\frac{216 x^{2} z^{2}-54 x y z^{2}-27 y^{2} z^{2}+27 y z^{2}-134 y z+188 y-54 z^{2}-108}{36 y(z-1)} \log \left(\frac{\mu^{2}}{M_{Z}^{2}}\right),
\end{aligned}
$$

with $x \equiv m_{t}^{2} / M_{Z}^{2}, y \equiv M_{h}^{2} / M_{Z}^{2}$, and $z=M_{Z}^{2} / M_{W}^{2}$. (Note that, again, a finite threshold correction has been included in order to express the result in terms of the five-flavor $\alpha$.) This result corresponds to the following definition of the relevant evanescent operator:

$$
E_{\mu}=\left(\bar{\nu}_{\mu, L} \gamma^{\mu_{1}} \gamma^{\mu_{2}} \gamma^{\mu_{3}} \mu_{L}\right)\left(\bar{\nu}_{e, L} \gamma_{\mu_{1}} \gamma_{\mu_{2}} \gamma_{\mu_{3}} e_{L}\right)-(16-4 \epsilon) Q_{\mu} .
$$

This form of the evanescent operator ensures the validity at $\mathcal{O}(\alpha)$ of the Fierz relations that have been used to calculate the QED corrections to the muon decay matrix element in the Fermi theory [21].

We can now discuss the different normalizations introduced in section 1, including the NLO corrections. For "GF" normalization we have

$$
c_{S 2}^{t t}=-\frac{1}{\sqrt{2} s_{w}^{2}} G_{\mathrm{F}} C_{S 2}^{\prime t t}=-\frac{2 \pi^{2}}{M_{W}^{2} s_{w}^{4}} C_{S 2}^{t t} .
$$


Inserting the all-order relation $G_{\mathrm{F}}=\pi \alpha /\left(\sqrt{2} M_{W}^{2} s_{w}^{2}\right) \times G_{\mu}$ and expanding $G_{\mu}$, we find

$$
\frac{\alpha}{4 \pi} C_{S 2}^{\prime t t}=C_{S 2}^{t t,(0)}+\frac{\alpha}{4 \pi}\left[C_{S 2}^{t t,(s e)}-C_{S 2}^{t t,(0)} G_{\mu}^{(s e)}\right]+\ldots
$$

Similarly, for "GF2" normalization we have

$$
c_{S 2}^{t t}=-\frac{M_{W}^{2}}{4 \pi^{2}} G_{\mathrm{F}}^{2} C_{S 2}^{\prime \prime t t}=-\frac{2 \pi^{2}}{M_{W}^{2} s_{w}^{4}} C_{S 2}^{t t}
$$

or

$$
\left(\frac{\alpha}{4 \pi}\right)^{2} C_{S 2}^{\prime \prime t t}=C_{S 2}^{t t,(0)}+\frac{\alpha}{4 \pi}\left[C_{S 2}^{t t,(s e)}-2 C_{S 2}^{t t,(0)} G_{\mu}^{(s e)}\right]+\ldots
$$

(Recall that we have absorbed factors of $\alpha /(4 \pi)$ into the definitions of the operators.) These relations allow us to obtain $C_{S 2}^{\prime t t}$ and $C_{S 2}^{\prime \prime t t}$ from our explicit calculations of $C_{S 2}^{t t}$ and $G_{\mu}$.

\section{Discussion of electroweak renormalization schemes}

The purpose of this section is to identify the residual theory uncertainty with regard to the higher-order electroweak corrections. We will estimate the uncertainty by studying different renormalization schemes for the input parameters, and the residual matching scale dependence in the $\overline{\mathrm{MS}}$ scheme. The immediate problem facing us is the dominant residual scale dependence with regard to QCD. In order to isolate the electroweak effects, we will therefore completely ignore QCD in this section. We define a formally scale- and scheme-independent quantity by multiplying the Wilson coefficient with the matrix element of the $Q_{S 2}$ operator. This serves to cancel the part of the scale dependence related to the anomalous dimension of the Wilson coefficient, and to cancel its residual scheme dependence (see the detailed discussion in section 4). We do not include any other finite terms in the matrix element. In principle, this matrix element could be calculated, including QCD effects, using lattice methods. The absolute value of the matrix element plays no role in our numerics, as in this section we are only interested in the scale and scheme variation, not the absolute values for the Wilson coefficients. These will be considered, including QCD, in the next section. To be specific, we multiply the Wilson coefficient by the partonic matrix element

$$
\left\langle Q_{S 2}\right\rangle \equiv\left[1+\frac{\alpha}{4 \pi} r^{(s e)}+\ldots\right]\left\langle Q_{S 2}\right\rangle^{(0)},
$$

where $\left\langle Q_{S 2}\right\rangle^{(0)}$ denotes the tree-level matrix element, and the LO QED correction is given by

$$
r^{(s e)}=\frac{1}{9} a_{11}-\frac{4}{3} \log \frac{\mu_{t}}{\mu_{\mathrm{had}}},
$$

with $\mu_{\text {had }}=2 \mathrm{GeV}$.

\section{1 $\overline{\mathrm{MS}}$ scheme}

Our primary input parameters in the $\overline{\mathrm{MS}}$ scheme are

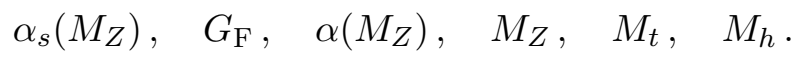


Their values are collected in table 1 . In the $\overline{\mathrm{MS}}$ scheme it is most convenient to express all physical quantities in terms of the running parameters

$$
g_{1}, g_{2}, g_{s}, y_{t}, \lambda, \quad v .
$$

To determine the initial conditions of these couplings at $\mu=M_{Z}$, we first use RunDec [22] to convert the top-quark pole mass to QCD- $\overline{\mathrm{MS}}$ using three-loop accuracy. We find $m_{t}\left(m_{t}\right)=$ 162.9(7) GeV. We then convert the (electroweak) on-shell masses $M_{Z}, m_{t}\left(m_{t}\right), M_{h}$ to $\overline{\mathrm{MS}}$ masses, using the one-loop expressions from [23, 24], and choosing the matching scale to be the respective on-shell masses. We take into account the correction (2.15) when converting $G_{\mathrm{F}}$ to $v$. We then fit the initial conditions of the couplings (3.4) such that they reproduce the central values of the converted input parameters at their respective scales, employing two-loop running of the couplings. Note that the strong coupling $g_{s}$ is neglected throughout this section (apart from the conversion of the top-quark mass to QCD- $\overline{\mathrm{MS}}$ ).

The two-loop beta functions for the SM are taken from ref. [25]. However, their result for the running of the Higgs vacuum expectation value is not consistent with our treatment of tadpole graphs (in fact, the result for $\beta_{v}$ given in ref. [25] is only valid in Landau gauge). A gauge-independent result consistent with our conventions can be extracted from refs. [23, 24]. For convenience, we collect the corresponding beta function here, writing $\beta_{v}=\beta_{v}^{(1)}+\beta_{v}^{(2)}+\ldots$ We find

$$
\beta_{v}^{(1)}=\frac{1}{16 \pi^{2}}\left(-\frac{3}{2} \lambda+\frac{3}{4} g_{1}^{2}+\frac{9}{4} g_{2}^{2}-\frac{3 g_{1}^{4}}{4 \lambda}-\frac{3 g_{1}^{2} g_{2}^{2}}{2 \lambda}-\frac{9 g_{2}^{4}}{4 \lambda}-3 y_{t}^{2}+12 \frac{y_{t}^{4}}{\lambda}\right),
$$

where we counted the contributions of colored fermions in ref. [24] with multiplicity three. As a check, we obtained the identical result by an explicit calculation of the one-loop renormalization of the Higgs mass, and using the beta function for the Higgs quartic from ref. [25] (see also ref. [26]). As a further consistency check, we note that $\beta_{v}^{(1)}$ is identical to the coefficient of the $\log \left(\mu^{2}\right)$ term in eq. (2.15), after expressing the mass ratios in terms of couplings. Also the two-loop beta function can be extracted from refs. [23, 24], as a combination of the beta functions for the $W$ mass and the $g_{2}$ gauge coupling. This yields

$$
\begin{aligned}
\beta_{v}^{(2)}=\frac{1}{\left(16 \pi^{2}\right)^{2}}( & \frac{1}{32} g_{2}^{4}-\frac{63}{16} g_{1}^{2} g_{2}^{2}-\frac{701}{96} g_{1}^{4}+\frac{379}{24} \frac{g_{1}^{6}}{\lambda}+\frac{559}{24} \frac{g_{1}^{4} g_{2}^{2}}{\lambda} \\
& +\frac{289}{24} \frac{g_{1}^{2} g_{2}^{4}}{\lambda}-\frac{305}{8} \frac{g_{2}^{6}}{\lambda}-\frac{3}{2} g_{1}^{2} \lambda-\frac{9}{2} g_{2}^{2} \lambda+\frac{63}{8} \lambda^{2} \\
& -\frac{85}{24} g_{1}^{2} y_{t}^{2}-\frac{45}{8} g_{2}^{2} y_{t}^{2}-\frac{21}{4} y_{t}^{4}+\frac{19}{2} \frac{g_{1}^{4} y_{t}^{2}}{\lambda} \\
& \left.-21 \frac{g_{1}^{2} g_{2}^{2} y_{t}^{2}}{\lambda}+\frac{9}{2} \frac{g_{2}^{4} y_{t}^{2}}{\lambda}+\frac{16}{3} \frac{g_{1}^{2} y_{t}^{4}}{\lambda}-60 \frac{y_{t}^{6}}{\lambda}+9 y_{t}^{2} \lambda-12 g_{2}^{2} g_{s}^{2}\right) .
\end{aligned}
$$

Part of the matching scale dependence of the Wilson coefficient cancels the scale dependence of the running parameters in the prefactor of the Lagrangian. Hence, we define a formally scale and scheme independent Wilson coefficient in the $\overline{\mathrm{MS}}$ scheme (where we 


\begin{tabular}{|l|l|}
\hline$M_{Z}=91.1876(21) \mathrm{GeV}$ & $M_{t}^{\text {pole }}=172.5(7) \mathrm{GeV}$ \\
\hline$M_{W}=80.379(12) \mathrm{GeV}$ & $\sin ^{2} \theta_{w}^{\overline{\mathrm{MS}}}=0.23141(4)$ \\
\hline$\alpha_{s}\left(M_{Z}\right)=0.1179(10)$ & $\alpha\left(M_{Z}\right)^{-1}=127.952(9)$ \\
\hline$G_{\mathrm{F}}=1.1663787(6) \times 10^{-5} \mathrm{GeV}^{-2}$ & $M_{h}=125.25(17) \mathrm{GeV}$ \\
\hline
\end{tabular}

Table 1. Primary input values used in our numerics; all numbers are taken from the PDG [2].
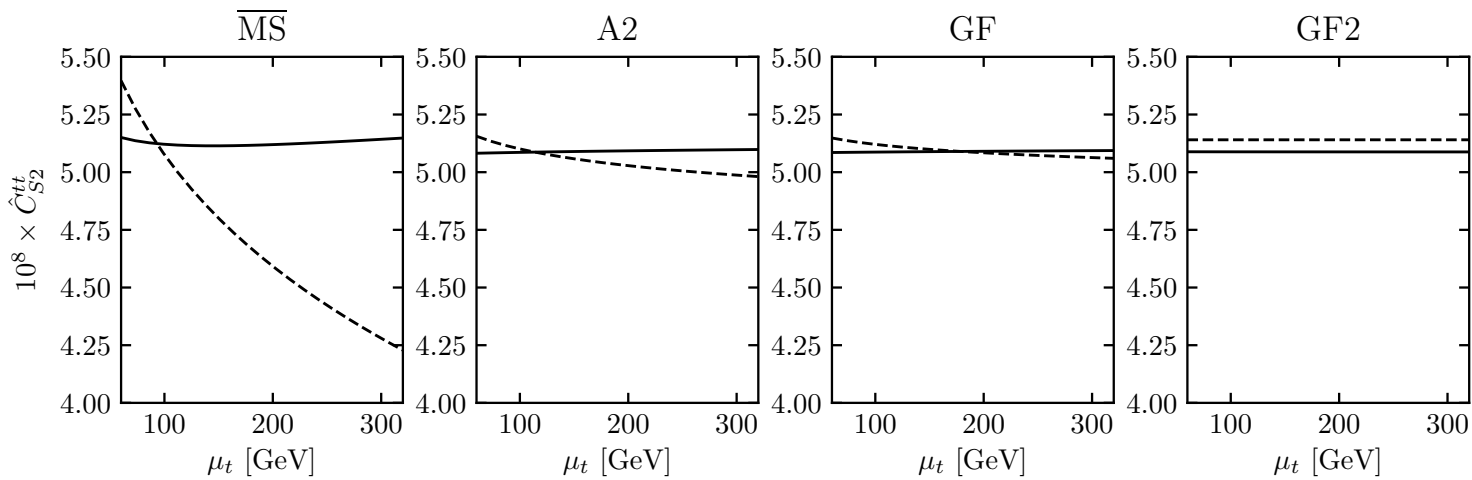

Figure 3. Residual electroweak matching scale dependence in the $\overline{\mathrm{MS}}$ and hybrid schemes. The dashed line shows the LO result, while the solid line show the NLO result. See text for details.

always use "A2" normalization)

$$
\hat{C}_{S 2}^{t t}=\frac{\alpha^{2}(\mu)}{8 m_{W}^{2}(\mu)\left(s_{w}^{\overline{\mathrm{MS}}}(\mu)\right)^{4}} C_{S 2}^{t t}(\mu)\left\langle Q_{S 2}\right\rangle(\mu)
$$

where $\alpha, m_{W}$, and $s_{w}^{\overline{\mathrm{MS}}}$ are defined in the $\overline{\mathrm{MS}}$ scheme, and the matrix element is defined in eq. (3.1). Varying the matching scale $\mu_{t}$ between $60 \mathrm{GeV}$ and $320 \mathrm{GeV}$, we see that the matching scale dependence is reduced from $\pm 12 \%$ at LO to $\pm 0.4 \%$ at NLO (see figure 3, left panel).

\subsection{On-shell scheme}

Here, we define all parameters in the on-shell scheme (OS) regarding the electroweak interaction. (We always treat the top-quark mass as an $\overline{\mathrm{MS}}$ mass regarding the strong interaction.) The weak mixing angle is defined, in the on-shell scheme, by $\sin ^{2} \theta_{w}^{\mathrm{OS}}=1-M_{W}^{2} / M_{Z}^{2}$. The $W$-boson mass itself is a function of the primary input parameters; to obtain its numerical value $M_{W}=80.354 \mathrm{GeV}$, we use the results of ref. [27]. Again, we define a formally scale- and scheme-independent Wilson coefficient. Its explicit form depends on the chosen normalization and is given by

$$
\hat{C}_{S 2}^{t t}=\frac{\alpha^{2}(\mu)}{8 M_{W}^{2}\left(s_{w}^{\mathrm{OS}}\right)^{4}} C_{S 2}^{t t}(\mu)\left\langle Q_{S 2}\right\rangle(\mu)
$$

for "A2" normalization, by

$$
\hat{C}_{S 2}^{t t}=\frac{\alpha(\mu) G_{\mathrm{F}}}{4 \sqrt{2}\left(s_{w}^{\mathrm{OS}}\right)^{2}} C_{S 2}^{\prime t t}(\mu)\left\langle Q_{S 2}\right\rangle(\mu)
$$



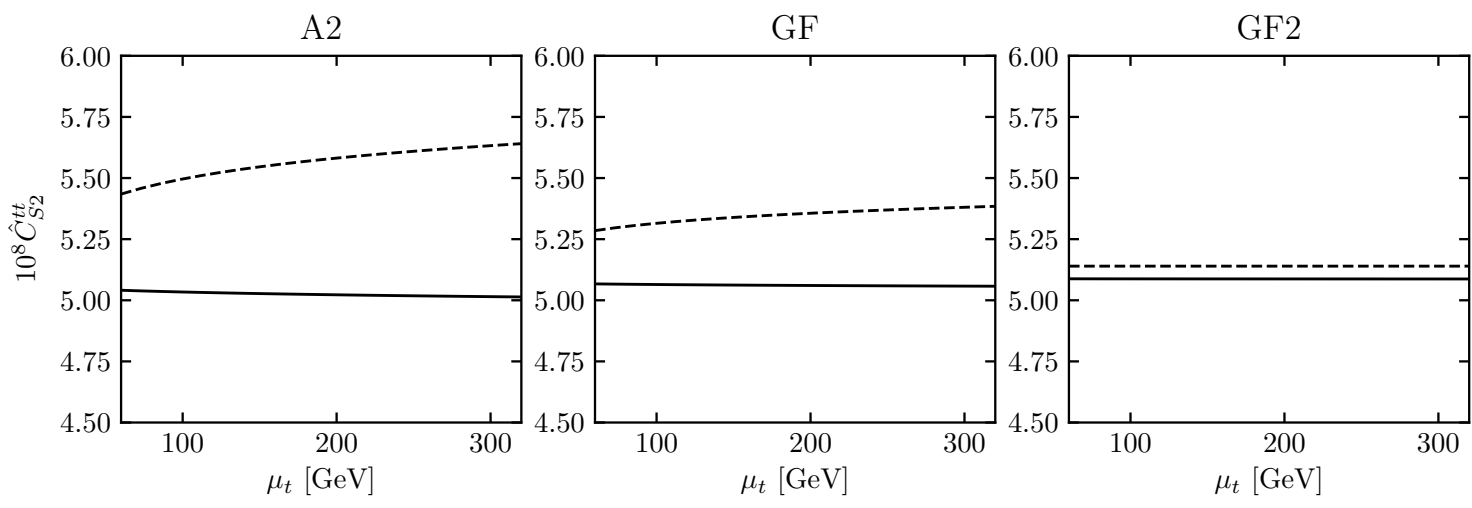

Figure 4. Residual electroweak matching scale dependence in the full OS scheme. The dashed line shows the LO result, while the solid line show the NLO result. See text for details.

for "GF" normalization, and by

$$
\hat{C}_{S 2}^{t t}=\frac{G_{\mathrm{F}}^{2} M_{W}^{2}}{4 \pi^{2}} C_{S 2}^{\prime \prime t t}(\mu)\left\langle Q_{S 2}\right\rangle(\mu)
$$

for "GF2" normalization. Here, $s_{w}^{\mathrm{OS}}$ and $M_{W}$ are defined in the on-shell scheme, while $\alpha$ is (always) defined as a $\overline{\mathrm{MS}}$ coupling (note that the dependence on $s_{w}^{\mathrm{OS}}$ drops out in "GF2" normalization).

As is clearly visible in figure 4, the NLO corrections in the on-shell scheme are large, indicating slow convergence of the perturbation series. As discussed in ref. [14], this can be attributed to the large top-mass dependence of the on-shell counterterm for the weak mixing angle. Hence, the on-shell scheme is not suitable for low-energy observables like $\epsilon_{K}$, and we will not use it in our error estimate.

\subsection{Hybrid scheme}

In this scheme, we define all masses in the on-shell scheme regarding the electroweak interaction, while the weak mixing angle that appears in the prefactor is defined in the $\overline{\mathrm{MS}}$ scheme: $\sin ^{2} \theta_{w}^{\overline{\mathrm{MS}}}=g_{1}^{2} /\left(g_{1}^{2}+g_{2}^{2}\right)$. Accordingly, we choose a different set of primary input parameters for the numerics in this scheme, namely,

$$
\alpha_{s}\left(M_{Z}\right), \quad \sin ^{2} \theta_{w}, \quad \alpha\left(M_{Z}\right), \quad M_{W}, \quad M_{t}, \quad M_{h} .
$$

Again, we fit the initial conditions of the $\overline{\mathrm{MS}}$ parameters (3.4) as described in section 3.1, neglecting the strong interaction. We define formally scale- and scheme-independent Wilson coefficients in this scheme as follows. For "A2" normalization,

$$
\hat{C}_{S 2}^{t t}=\frac{\alpha^{2}(\mu)}{8 M_{W}^{2}\left(s_{w}^{\overline{\mathrm{MS}}}(\mu)\right)^{4}} C_{S 2}^{t t}(\mu)\left\langle Q_{S 2}\right\rangle(\mu),
$$

while for "GF" normalization,

$$
\hat{C}_{S 2}^{t t}=\frac{\alpha(\mu) G_{\mathrm{F}}}{4 \sqrt{2}\left(s_{w}^{\overline{\mathrm{MS}}}(\mu)\right)^{2}} C_{S 2}^{\prime t t}(\mu)\left\langle Q_{S 2}\right\rangle(\mu) .
$$


Here, $s_{w}^{\overline{\mathrm{MS}}}(\mu)$ is defined in the $\overline{\mathrm{MS}}$ scheme, while $M_{W}$ is still defined in the on-shell scheme. Note that the hybrid scheme coincides with the on-shell scheme for the "GF2" normalization, up to the tiny numerical difference in the $M_{W}$ mass.

The Wilson coefficients for the three different normalizations are shown in figure 3 (right three panels). While the LO results in "A2" and "GF" normalization still show sizeable scale dependence, all NLO curves are essentially flat. As an additional estimate of the unknown higher-order electroweak effects, we take half of the absolute value of the difference between the largest and the smallest NLO value. Again, this translates into a $\pm 0.4 \%$ uncertainty.

\section{Inclusion of QCD}

As is well-known, the dominant corrections to the LO SM prediction of the $|\Delta S|=2$ weak Lagrangian arise from QCD and have to be taken into account in the final numerics. In this section, we will summarize the status of the QCD corrections and combine them with our electroweak corrections.

The large separation between the electroweak scale, $\mu_{t} \sim m_{t}, M_{W}$ and the scale $\mu_{\text {had }} \sim$ $2 \mathrm{GeV}$ where the hadronic matrix elements are evaluated mandate the use of RG-improved perturbation theory, summing powers of $\alpha_{s} \log \left(\mu_{t} / \mu_{\text {had }}\right)$ to all orders. Here, we include also the (small) QED corrections. The formalism is well-known $[6,28]$. We now expand the Wilson coefficient, including the QCD terms, as

$$
C_{S 2}^{t t}(\mu)=C_{S 2}^{t t,(0)}(\mu)+\frac{\alpha_{s}(\mu)}{4 \pi} C_{S 2}^{t t,(1)}(\mu)+\frac{\alpha}{\alpha_{s}(\mu)} C_{S 2}^{t t,(e)}(\mu)+\frac{\alpha}{4 \pi} C_{S 2}^{t t,(s e)}(\mu)+\ldots
$$

The initial conditions $C_{S 2}^{t t,(0)}\left(\mu_{t}\right)$ and $C_{S 2}^{t t,(s e)}\left(\mu_{t}\right)$ have been given in the previous section. The coefficient $C_{S 2}^{t t,(e)}\left(\mu_{t}\right)$ does not receive a matching contribution; it is purely generated by the RG flow and corresponds to LL QED resummed logarithms of the form $\alpha \alpha_{s}^{n} \log ^{n+1}\left(\mu_{t} / \mu_{\text {had }}\right)$. The last term contains the summed NLL QED logarithms of the form $\alpha \alpha_{s}^{n} \log ^{n}\left(\mu_{t} / \mu_{\text {had }}\right)$. The coefficient $C_{S 2}^{t t,(1)}\left(\mu_{t}\right)$ receives matching contributions from two-loop box diagrams with gluon exchange. As a check of our setup we calculated this term and find

$$
\begin{aligned}
C_{S 2}^{t t,(1)}(\mu)= & \frac{8 \pi^{2}}{9 x_{t}}+\frac{10 x_{t}^{5}+55 x_{t}^{4}+149 x_{t}^{3}+4 x_{t}^{2}}{6\left(x_{t}-1\right)^{4}} \log ^{2}\left(x_{t}\right) \\
& +\frac{-15 x_{t}^{5}-2 x_{t}^{4}-543 x_{t}^{3}+388 x_{t}^{2}-148 x_{t}+32}{6\left(x_{t}-1\right)^{4}} \log \left(x_{t}\right) \\
& +\frac{25 x_{t}^{4}+248 x_{t}^{3}+75 x_{t}^{2}-92 x_{t}+32}{6\left(x_{t}-1\right)^{3}} \\
& +\operatorname{Li}_{2}\left(1-x_{t}\right) \frac{10 x_{t}^{5}+74 x_{t}^{4}-2 x_{t}^{3}+40 x_{t}^{2}-48 x_{t}+16}{3 x_{t}\left(x_{t}-1\right)^{3}} \\
& +\frac{5 x_{t}^{4}+87 x_{t}^{2}-20 x_{t}}{2\left(x_{t}-1\right)^{3}} \log \left(x_{\mu}\right)+\frac{3 x_{t}^{4}-39 x_{t}^{3}}{\left(x_{t}-1\right)^{4}} \log \left(x_{t}\right) \log \left(x_{\mu}\right),
\end{aligned}
$$



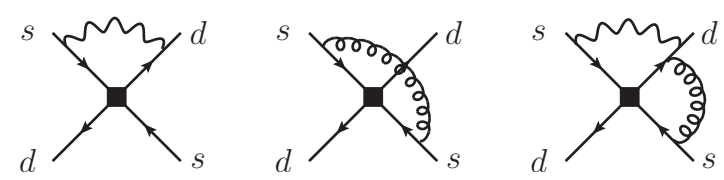

Figure 5. Sample Feynman diagrams contributing to the two-loop anomalous dimension.

where $x_{\mu} \equiv \mu^{2} / M_{W}^{2}$, and $\operatorname{Li}_{2}(x)=-\int_{0}^{x} d u \ln (1-u) / u$ is the usual dilogarithm. This expression is in full agreement the well-known result presented in ref. [8].

The RG evolution is most conveniently written in terms of an evolution matrix, such that $C_{S 2}^{t t}(\mu)=C_{S 2}^{t t}\left(\mu_{0}\right) U\left(\mu_{0}, \mu, \alpha\right)$. We expand

$$
U\left(\mu_{0}, \mu, \alpha\right)=U^{(0)}\left(\mu_{0}, \mu\right)+\frac{\alpha_{s}(\mu)}{4 \pi} U^{(1)}\left(\mu_{0}, \mu\right)+\frac{\alpha}{\alpha_{s}(\mu)} U^{(e)}\left(\mu_{0}, \mu\right)+\frac{\alpha}{4 \pi} U^{(s e)}\left(\mu_{0}, \mu\right)
$$

in terms of the couplings defined at the low scale $\mu$, and find the following contributions to the Wilson coefficient at the low scale:

$$
\begin{aligned}
C_{S 2}^{t t,(0)}(\mu)= & C_{S 2}^{t t,(0)}\left(\mu_{0}\right) U^{(0)}\left(\mu_{0}, \mu\right), \\
C_{S 2}^{t t,(1)}(\mu)= & \eta C_{S 2}^{t t,(1)}\left(\mu_{0}\right) U^{(0)}\left(\mu_{0}, \mu\right)+C_{S 2}^{t t,(0)}\left(\mu_{0}\right) U^{(1)}\left(\mu_{0}, \mu\right), \\
C_{S 2}^{t t,(e)}(\mu)= & C_{S 2}^{t t,(0)}\left(\mu_{0}\right) U^{(e)}\left(\mu_{0}, \mu\right)+\eta^{-1} C_{S 2}^{t t,(e)}\left(\mu_{0}\right) U^{(0)}\left(\mu_{0}, \mu\right), \\
C_{S 2}^{t t,(s e)}(\mu)= & \eta C_{S 2}^{t t,(1)}\left(\mu_{0}\right) U^{(e)}\left(\mu_{0}, \mu\right)+\eta^{-1} C_{S 2}^{t t,(e)}\left(\mu_{0}\right) U^{(1)}\left(\mu_{0}, \mu\right) \\
& +C_{S 2}^{t t,(s e)}\left(\mu_{0}\right) U^{(0)}\left(\mu_{0}, \mu\right)+C_{S 2}^{t t,(0)}\left(\mu_{0}\right) U^{(s e)}\left(\mu_{0}, \mu\right),
\end{aligned}
$$

where we have introduced the ratio $\eta=\alpha_{s}\left(\mu_{0}\right) / \alpha_{s}(\mu)$. The explicit expression for the evolution matrix can be found in ref. [28] and involves the anomalous dimension of the Wilson coefficients. It is given by

$$
\gamma_{S 2}^{(0)}=\frac{\alpha_{s}}{\pi}+\left(\frac{4 N_{f}}{9}-7\right) \frac{\alpha_{s}^{2}}{16 \pi^{2}}+\frac{\alpha}{3 \pi}-\frac{148}{9} \frac{\alpha \alpha_{s}}{16 \pi^{2}} .
$$

The first two terms are well-known [6], while the QED corrections are new. They have been calculated by extracting the UV poles of the relevant one- and two-loop diagrams (see figure 5 for examples) using the infrared rearrangement described in ref. [29].

The result $(4.8)$ is valid for the Wilson coeffients $\left(C_{S 2}^{t t}, C_{S 2}^{\prime t t}\right.$, and $\left.C_{S 2}^{\prime \prime t t}\right)$ in all three normalization conventions, due to our absorbing powers of $\alpha$ into the definitions of the corresponding operators. Note that the $\alpha_{s}^{2}$ term depends on the explicit form of the evanescent operators, given in appendix B, while the other three terms are scheme independent, as we have verified by explicit calculation.

This last observation deserves further discussion. Our two-loop result $C_{S 2}^{t t,(s e)}$ does depend on the definition of evanescent operators in eq. (B.1). The SM prediction for the observable $\epsilon_{K}$ must, of course, be independent of such arbitrary choices; in fact, the scheme dependence of the Wilson coefficient will cancel exactly against the corresponding scheme dependence of the hadronic matrix element (a proof is given in appendix $\mathrm{C}$ ). In the literature on $\epsilon_{K}$, the scheme independent product of Wilson coefficient and matrix element 

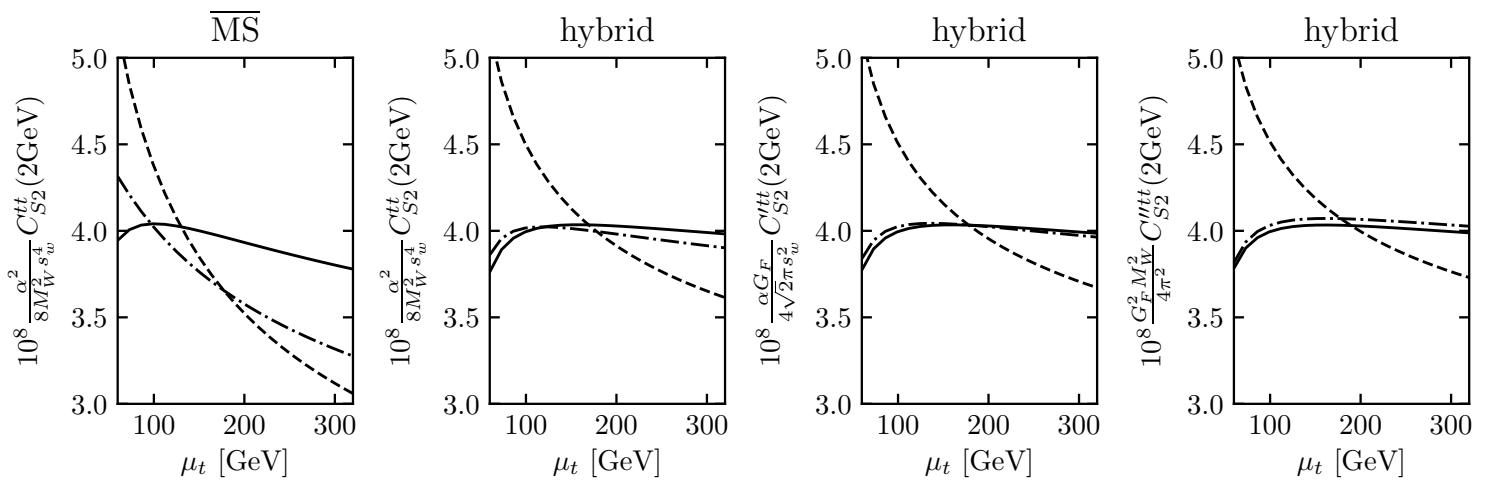

Figure 6. Residual matching scale dependence with full QCD resummation, in the $\overline{\mathrm{MS}}$ and hybrid schemes. The dashed line shows the LO result, the dash-dotted line shows the result including NLO QCD corrections, while the solid line show the full (QCD and QED) NLO result. See text for details.

is usually factorized into two separately scheme- and scale-independent quantities, namely, the QCD correction factors $\eta_{t t}$ and $\eta_{u t}$, and the kaon bag factor $\hat{B}_{K}$. This is achieved by writing the evolution matrix as $U\left(\mu_{0}, \mu, \alpha\right)=K^{-1}\left(\mu_{0}, \alpha\right) U^{(0)}\left(\mu_{0}, \mu, \alpha\right) K(\mu, \alpha)$, and combining the $K$ factors, together with the appropriate part of the LO evolution matrix, with the Wilson coefficients and the matrix elements to yield scheme-independent quantities (see refs. [6, 28] for details).

In our case, this strategy fails when including QED corrections, as the $\mathcal{O}\left(\alpha \alpha_{s}\right)$ anomalous dimension is scheme independent by itself. This is consistent with the general expression for the scheme dependence of anomalous dimensions given in ref. [28] and appendix C, because here the anomalous dimension is a one-dimensional matrix, i.e. just a number. Therefore, the definition of the scheme-invariant correction factor $\eta_{t t}$ cannot be extended to include QED effects (as a fixed-order expansion in $\alpha$ ).

It follows that, in the absence of a determination of the hadronic matrix element including QED corrections, our result will be scheme dependent. However, the scheme dependence is tiny. Numerically, the explicit dependence on the coefficient $a_{11}$, defined in appendix $\mathrm{B}$, is

$$
C_{S 2}^{\prime \prime t t}(2 \mathrm{GeV})=\left(3.90-0.0003 a_{11}\right) \times 10^{-8} .
$$

This dependence will cancel once a full matrix element is available. However, we expect the finite shift in the hadronic matrix element to be equally tiny, of order $\alpha /(4 \pi) \sim 10^{-4}$. The bulk of the effect of electroweak corrections is captured by the matching calculation at the weak scale, not by the QED effects in the effective theory. We will therefore neglect the scheme dependence in our numerical discussion.

To quantify the impact of the electroweak corrections, we examine the numerical values of the Wilson coefficients in the hybrid renormalization scheme, as it shows the best convergence properties and least residual scale dependence (cf. figure 6). The values in the three different normalization conventions are given in table 2, both for the NLL QCD result only, and including the full NLO electroweak corrections (with NLL QED resummation). 


\begin{tabular}{|l|c|c|}
\hline & NLL QCD & NLL QCD \& NLL QED \\
\hline$\alpha^{2} /\left(8 M_{W}^{2} s_{w}^{4}\right) C_{S 2}^{t t}(2 \mathrm{GeV}) \times 10^{8}$ & $3.96(6)$ & $3.98(6)$ \\
\hline$\alpha G_{\mathrm{F}} /\left(4 \sqrt{2} \pi s_{w}^{2}\right) C_{S 2}^{\prime t t}(2 \mathrm{GeV}) \times 10^{8}$ & $4.00(4)$ & $3.98(5)$ \\
\hline$G_{\mathrm{F}}^{2} M_{W}^{2} /\left(4 \pi^{2}\right) C_{S 2}^{\prime \prime t}(2 \mathrm{GeV}) \times 10^{8}$ & $4.02(5)$ & $3.98(5)$ \\
\hline
\end{tabular}

Table 2. Wilson coefficients. Uncertainty given is scale variation only.

The shown uncertainties are half of the difference between maximum and minimum values when varying the matching scale $\mu_{t}$ between $60 \mathrm{GeV}$ and $320 \mathrm{GeV}$. We see that the shift from NLL QCD to full electroweak is actually largest, $-1 \%$, in the conventionally used "GF2" normalization, while in the "A2" and "GF" normalizations the shift is $+0.5 \%$ and $-0.5 \%$, respectively. ${ }^{3}$ The resulting NLO values are in perfect agreement.

With the three-loop QCD corrections in the top sector and the electroweak corrections in the charm-top sector still outstanding, we refrain from an extensive numerical discussion of our results. The shift in the Wilson coefficient is small, of order one percent, in the hybrid scheme in all normalizations. Since both the residual scale dependence and the spread between NLO values (considering electroweak effects only) is both $\pm 0.4 \%$ (see section 3 ), we suggest the following temporary prescription that is most easily implemented: adopt the traditional "GF2" normalization, and multiply $\eta_{t t}$ by the electroweak correction factor $\left(1-\Delta_{t t}\right)$, with $\Delta_{t t}=0.01 \pm 0.004$. With this prescription, the SM prediction presented in ref. [7] is shifted by $-0.7 \%$ to $\left|\epsilon_{K}\right|=2.15(6)(7)(15) \times 10^{-3}$, with the errors corresponding to short-distance, long-distance, and parametric uncertainties (see ref. [7] for details).

\section{Conclusions}

We have presented the complete two-loop electroweak corrections to the top-quark contribution to the parameter $\epsilon_{K}$. The analogous electroweak corrections for $B^{0}-\overline{B^{0}}$ mixing have been presented previously in ref. [13]; as a check, we reproduced their numerical results using the input parameters given in that reference. In our calculation we used three different normalizations for the effective Lagrangian and several renormalization schemes for the electroweak input parameters. While these lead to different numerical predictions at LO, the final results agree well if the electroweak corrections are taken into account. We assess the theoretical uncertainty by studying the residual electroweak matching scale dependence and the residual dependence on the renormalization scheme for the input parameters, leading to an error of $\pm 0.4 \%$ associated with unknown higher-order corrections.

We then considered the full RG evolution in the effective theory at NLO in QCD and mixed QED-QCD. In particular, we discuss how the scheme dependence, related to the freedom in choosing the evanescent operators, cancels against the hadronic matrix element if evaluated including QED corrections. While these corrections are currently unknown, they are expected to be tiny, as is the leftover scheme dependence of our result.

\footnotetext{
${ }^{3}$ Using the (even) more conservative range $40 \mathrm{GeV} \leq \mu_{t} \leq 320 \mathrm{GeV}$ leads to a $-1 \%$ shift in all three normalizations.
} 
Numerically, the inclusion of the electroweak corrections amounts to a $-1 \%$ downward shift in the Wilson coefficient for the top-quark contribution defined at the hadronic scale $\mu_{\text {had }}=2 \mathrm{GeV}$, when using the conventional normalization of the effective Lagrangian. We defer a more extensive numerical study of the effect on the SM prediction of $\epsilon_{K}$ to the future, when the three-loop QCD corrections in the top sector [12], the two-loop electroweak corrections in the charm-top sector [30], and possibly an updated hadronic matrix element will be available. Instead, we just shift the usual QCD correction factor $\eta_{t t}$ by a factor $1-0.010(4)$. This shifts the SM prediction presented in ref. [7] by $-0.7 \%$ to $\left|\epsilon_{K}\right|=2.15(6)(7)(15) \times 10^{-3}$. The errors correspond to short-distance, long-distance, and parametric uncertainties.

\section{Acknowledgments}

We thank Jens Erler for useful communication regarding the electroweak input parameters, and Martin Gorbahn and Emmanuel Stamou for many discussions and comments on the manuscript. JB acknowledges support by DOE grant DE-SC0011784. The Feynman diagrams have been generated using jaxodraw [31], based on axodraw [32].

\section{A Mass and field renormalization constants}

All mass renormalization constants include the tadpole contributions and are thus gaugeparameter independent:

$$
\begin{aligned}
& \delta M_{W}^{2}=\frac{e^{2}}{16 \pi^{2} \epsilon} M_{W}^{2}[ \frac{3}{4}-\frac{11}{12 s_{w}^{2}}+\frac{3}{4} \frac{s_{w}^{2}}{c_{w}^{2}}-\frac{3}{2 s_{w}^{2}} \frac{m_{t}^{2}}{M_{W}^{2}} \\
&\left.-\frac{3}{4 s_{w}^{2}} \frac{M_{h}^{2}}{M_{W}^{2}}+\frac{6}{s_{w}^{2}} \frac{m_{t}^{4}}{M_{W}^{2} M_{h}^{2}}-\frac{3}{2 s_{w}^{2} c_{w}^{4}} \frac{M_{W}^{2}}{M_{h}^{2}}-\frac{3}{s_{w}^{2}} \frac{M_{W}^{2}}{M_{h}^{2}}\right] \\
& \delta M_{Z}^{2}=\frac{e^{2}}{16 \pi^{2} \epsilon} M_{Z}^{2}\left[\frac{29}{6 c_{w}^{2}}-\frac{11}{12} \frac{1}{s_{w}^{2} c_{w}^{2}}+\frac{11}{3} \frac{s_{w}^{2}}{c_{w}^{2}}-\frac{3}{2 s_{w}^{2} c_{w}^{2}} \frac{m_{t}^{2}}{M_{Z}^{2}}\right.\left.-\frac{3}{4 s_{w}^{2} c_{w}^{2}} \frac{M_{h}^{2}}{M_{Z}^{2}}+\frac{6}{s_{w}^{2} c_{w}^{2}} \frac{m_{t}^{4}}{M_{Z}^{2} M_{h}^{2}}-\frac{3}{2 s_{w}^{2} c_{w}^{2}} \frac{M_{Z}^{2}}{M_{h}^{2}}-\frac{3}{s_{w}^{2}} \frac{M_{Z}^{2}}{M_{h}^{2}}+3 \frac{M_{Z}^{2}}{M_{h}^{2}}\right], \\
& \delta M_{h}^{2}=\frac{e^{2}}{16 \pi^{2} \epsilon} M_{h}^{2}\left[-\frac{3}{4 s_{w}^{2} c_{w}^{2}}-\frac{3}{2 s_{w}^{2}}+\frac{3}{4 s_{w}^{2}} \frac{M_{h}^{2}}{M_{W}^{2}}+\frac{3}{2 s_{w}^{2}} \frac{m_{t}^{2}}{M_{W}^{2}}\right] \\
& \delta m_{t}=\frac{e^{2}}{16 \pi^{2} \epsilon} m_{t}\left[-\frac{3}{4 s_{w}^{2} c_{w}^{2}} \frac{M_{Z}^{2}}{M_{h}^{2}}-\frac{3}{2 s_{w}^{2}} \frac{M_{W}^{2}}{M_{h}^{2}}-\frac{3}{8 s_{w}^{2}} \frac{M_{h}^{2}}{M_{W}^{2}}\right. \\
&\left.-\frac{1}{3 c_{w}^{2}}+\frac{3}{8 s_{w}^{2}} \frac{m_{t}^{2}}{M_{W}^{2}}+\frac{3}{s_{w}^{2}} \frac{m_{t}^{4}}{M_{W}^{2} M_{h}^{2}}\right]
\end{aligned}
$$

It follows that

$$
\frac{\delta s_{w}^{2}}{s_{w}^{2}}=-\frac{c_{w}^{2}}{s_{w}^{2}} \frac{\delta c_{w}^{2}}{c_{w}^{2}}=\frac{e^{2}}{16 \pi^{2} \epsilon}\left[\frac{11}{3}+\frac{19}{6 s_{w}^{2}}\right]
$$


Note that all tadpole contributions to $\delta s_{w}^{2}$ cancel. The charge renormalization is given by

$$
\delta Z_{e}=\frac{e^{2}}{16 \pi^{2} \epsilon}\left[\frac{8 N_{u}}{9}+\frac{2 N_{d}}{9}+\frac{2 N_{\ell}}{3}-\frac{7}{2}\right] .
$$

The divergent part of the one-loop off-diagonal field renormalization constant (in 't HooftFeynman gauge) is

$$
\delta Z_{s d}^{L}=-\frac{e^{2}}{16 \pi^{2} \epsilon} \frac{V_{t s} V_{t d}^{*}}{s_{w}^{2}} \frac{x_{t}}{4}
$$

The finite part of the one-loop off-diagonal field renormalization constant (in 't HooftFeynman gauge) is

$$
\delta Z_{s d}^{L}=-\frac{e^{2}}{16 \pi^{2}} \frac{V_{t s} V_{t d}^{*}}{s_{w}^{2}}\left[\frac{3\left(x_{t}^{2}+x_{t}\right)}{8\left(x_{t}-1\right)}+\frac{x_{t}-4 x_{t}^{2}}{4\left(x_{t}-1\right)^{2}} \log x_{t}+\frac{x_{t}}{4} \log \frac{\mu^{2}}{m_{t}^{2}}\right],
$$

where $x_{t} \equiv m_{t}^{2} / M_{W}^{2}$. Both eqs. (A.7) and (A.8) are in agreement with the results in ref. [13]. The divergent part of the one-loop diagonal field renormalization constant for the down-type quarks (in 't Hooft-Feynman gauge) is

$$
\delta Z_{d d}^{L}=-\frac{e^{2}}{16 \pi^{2} \epsilon} \frac{1}{s_{w}^{2}}\left[V_{t d} V_{t d}^{*} \frac{x_{t}}{4}+\frac{13}{18}+\frac{1}{36 c_{w}^{2}}\right] .
$$

The finite part of the one-loop diagonal field renormalization constant for the down-type quarks (in 't Hooft-Feynman gauge) is

$$
\begin{aligned}
\delta Z_{d d}^{L}= & -\frac{e^{2}}{16 \pi^{2}} \frac{V_{t d} V_{t d}^{*}}{s_{w}^{2}}\left[\frac{3\left(x_{t}^{2}+x_{t}\right)}{8\left(x_{t}-1\right)}+\frac{x_{t}-4 x_{t}^{2}}{4\left(x_{t}-1\right)^{2}} \log x_{t}+\frac{x_{t}}{4} \log \frac{\mu^{2}}{m_{t}^{2}}\right] \\
& +\frac{e^{2}}{16 \pi^{2} s_{w}^{2}}\left[\frac{11}{36}+\frac{1}{72 c_{w}^{2}}+\frac{c_{w}^{2}}{18}-\left(\left(1+c_{w}^{2}\right) \frac{1}{9}+\frac{1}{36 c_{w}^{2}}\right) \log x-\frac{1}{2} \log x_{t}\right] \\
& -\frac{e^{2}}{16 \pi^{2} s_{w}^{2}}\left[\frac{11}{18}+\frac{1}{36 c_{w}^{2}}+\frac{c_{w}^{2}}{9}\right] \log \frac{\mu^{2}}{m_{t}^{2}} .
\end{aligned}
$$

The finite part differs from the expression in ref. [13] due to our using dimensional regularization for the IR divergences.

\section{B Renormalization constants in the effective theory}

The evanescent operators necessary for the calculation of the matching conditions and anomalous dimensions up to two-loop in QCD and QED are chosen as [10]

$$
\begin{aligned}
& F_{S 2}^{(0)}=\left(\frac{\alpha}{4 \pi}\right)^{2}\left(\bar{s}_{L}^{\alpha} \gamma_{\mu} d_{L}^{\beta}\right) \otimes\left(\bar{s}_{L}^{\beta} \gamma^{\mu} d_{L}^{\alpha}\right)-Q_{S 2}, \\
& E_{S 2}^{(1)}=\left(\frac{\alpha}{4 \pi}\right)^{2}\left(\bar{s}_{L}^{\alpha} \gamma_{\mu_{1}} \gamma_{\mu_{2}} \gamma_{\mu_{3}} d_{L}^{\alpha}\right) \otimes\left(\bar{s}_{L}^{\beta} \gamma^{\mu_{1}} \gamma^{\mu_{2}} \gamma^{\mu_{3}} d_{L}^{\beta}\right)-\left(16-a_{11} \epsilon-4 \epsilon^{2}\right) Q_{S 2},
\end{aligned}
$$




$$
\begin{aligned}
F_{S 2}^{(1)}= & \left(\frac{\alpha}{4 \pi}\right)^{2}\left(\bar{s}_{L}^{\alpha} \gamma_{\mu_{1}} \gamma_{\mu_{2}} \gamma_{\mu_{3}} d_{L}^{\beta}\right) \otimes\left(\bar{s}_{L}^{\beta} \gamma^{\mu_{1}} \gamma^{\mu_{2}} \gamma^{\mu_{3}} d_{L}^{\alpha}\right)-\left(16-b_{11} \epsilon-4 \epsilon^{2}\right)\left(Q_{S 2}+F_{S 2}^{(0)}\right) \\
E_{S 2}^{(2)}= & \left(\frac{\alpha}{4 \pi}\right)^{2}\left(\bar{s}_{L}^{\alpha} \gamma_{\mu_{1}} \gamma_{\mu_{2}} \gamma_{\mu_{3}} \gamma_{\mu_{4}} \gamma_{\mu_{5}} d_{L}^{\alpha}\right) \otimes\left(\bar{s}_{L}^{\beta} \gamma^{\mu_{1}} \gamma^{\mu_{2}} \gamma^{\mu_{3}} \gamma^{\mu_{4}} \gamma^{\mu_{5}} d_{L}^{\beta}\right) \\
& -\left(256-a_{21} \epsilon-\frac{108816}{325} \epsilon^{2}\right) Q_{S 2} \\
F_{S 2}^{(2)}= & \left(\frac{\alpha}{4 \pi}\right)^{2}\left(\bar{s}_{L}^{\alpha} \gamma_{\mu_{1}} \gamma_{\mu_{2}} \gamma_{\mu_{3}} \gamma_{\mu_{4}} \gamma_{\mu_{5}} d_{L}^{\beta}\right) \otimes\left(\bar{s}_{L}^{\beta} \gamma^{\mu_{1}} \gamma^{\mu_{2}} \gamma^{\mu_{3}} \gamma^{\mu_{4}} \gamma^{\mu_{5}} d_{L}^{\alpha}\right) \\
& -\left(256-b_{21} \epsilon-\frac{108816}{325} \epsilon^{2}\right)\left(Q_{S 2}+F_{S 2}^{(0)}\right)
\end{aligned}
$$

The terms quadratic in $\epsilon$ do not play a role in our calculation and are kept only for completeness. Some of the coefficients of the $\epsilon$ terms have been left unspecified as an additional check of our calculation. The result for the Wilson coefficient at NLO in the electroweak interaction depends on the coefficient $a_{11}$, as discussed in section 4 . Our results given in this paper and all plots use the conventional choice $a_{11}=4$.

We expand the renormalization constants as

$$
Z=1+\sum_{k=0}^{\infty}\left[\sum_{i=1}^{\infty} Z^{(i, k)}\left(\frac{\alpha_{s}}{4 \pi}\right)^{i}+Z^{(e, k)} \frac{\alpha}{4 \pi}+Z^{(s e, k)} \frac{\alpha \alpha_{s}}{(4 \pi)^{2}}\right] \frac{1}{\epsilon^{k}} .
$$

The necessary, non-zero $Z$ factors at order $\alpha_{s}$ are

$$
Z_{Q_{S 2}, Q_{S 2}}^{(1,1)}=2, \quad Z_{Q_{S 2}, F_{S 2}^{(0)}}^{(1,1)}=3, \quad Z_{Q_{S 2}, E_{S 2}^{(1)}}^{(1,1)}=-\frac{1}{6}, \quad Z_{Q_{S 2}, F_{S 2}^{(1)}}^{(1,1)}=\frac{1}{2},
$$

and

$$
\begin{aligned}
& Z_{F_{S 2}^{(1)}, Q_{S 2}}^{(1,0)}=2-\frac{5}{12} a_{11}-\frac{1}{12} b_{11}, \quad Z_{E_{S 2}^{(1)}, Q_{S 2}}^{(1,0)}=-48-\frac{7}{3} a_{11}+13 b_{11}+\frac{1}{6} a_{21}-\frac{1}{2} b_{21}, \\
& Z_{F_{S 2}^{(1,0)}, Q_{S 2}}^{(1)}=48+5 a_{11}+\frac{65}{3} b_{11}-\frac{1}{4} a_{21}-\frac{7}{12} b_{21} .
\end{aligned}
$$

The necessary, non-zero $Z$ factors at order $\alpha$ are

$$
Z_{Q_{S 2}, Q_{S 2}}^{(e, 1)}=\frac{2}{3}, \quad Z_{Q_{S 2}, E_{S 2}^{(1)}}^{(e, 1)}=\frac{1}{9},
$$

and

$$
Z_{F_{S 2}^{(0)}, Q_{S 2}}^{(e, 0)}=\frac{1}{9} a_{11}-\frac{1}{9} b_{11}, \quad Z_{E_{S 2}^{(1)}, Q_{S 2}}^{(e, 0)}=\frac{32}{9} a_{11}-\frac{1}{9} a_{21}, \quad Z_{F_{S 2}^{(1)}, Q_{S 2}}^{(e, 0)}=\frac{32}{9} b_{11}-\frac{1}{9} b_{21} .
$$

At two-loop, we find

$$
Z_{Q_{S 2}, Q_{S 2}}^{(s e, 1)}=-\frac{61}{9}-\frac{7}{27} a_{11}+\frac{13}{9} b_{11}+\frac{1}{54} a_{21}-\frac{1}{18} b_{21} .
$$

Using these renormalization constants, we find that all scheme dependence cancels in the two-loop anomalous dimension at order $\alpha \alpha_{s}$, as expected on general grounds [28]. 


\section{Scheme independence at order $\alpha$}

In this appendix we give a proof of the scheme independence of the prediction of the topquark contribution to $\epsilon_{K}$ at NLO in QED. We first recall the transformation properties of Wilson coefficients and anomalous dimension at order $\alpha$ by adapting the results in ref. [10]. To this end, we write the general transformation among all dimension-six operators as

$$
\left(\begin{array}{l}
Q^{\prime} \\
E^{\prime}
\end{array}\right)=\left(\begin{array}{cc}
R & 0 \\
0 & M
\end{array}\right)\left(\begin{array}{cr}
1 & 0 \\
\epsilon U+\epsilon^{2} V & 1
\end{array}\right)\left(\begin{array}{ll}
1 & W \\
0 & 1
\end{array}\right)\left(\begin{array}{l}
Q \\
E
\end{array}\right) .
$$

Here, the matrices $R$ and $M$ parameterize a linear transformation among the physical and evanescent operators $Q$ and $E$, respectively, $W$ parameterizes the addition of multiples of evanescent operators to the physical operators, and $U$ parameterizes the addition of multiples of $\epsilon$ times physical operators to the evanescent operators. As explained in detail in ref. [33], this transformation implies an additional finite renormalization that is needed in order to restore the standard $\overline{\mathrm{MS}}$ definition of the renormalization constants. This finite renormalization, induced by the change (C.1), is given in the notation of the previous section by

$$
\begin{aligned}
& Z_{Q Q}^{(1,0)}=R\left[W Z_{E Q}^{(1,0)}-\left(Z_{Q E}^{(1,1)}+W Z_{E E}^{(1,1)}-\frac{1}{2} \gamma^{(0)} W\right) U\right] R^{-1}, \\
& Z_{Q Q}^{(e, 0)}=R\left[W Z_{E Q}^{(e, 0)}-\left(Z_{Q E}^{(e, 1)}+W Z_{E E}^{(e, 1)}-\frac{1}{2} \gamma^{(e)} W\right) U\right] R^{-1},
\end{aligned}
$$

by a straightforward generalization of the results in ref. [10], where also the higher-order QCD expressions can be found. The corresponding transformation law for the anomalous dimension matrices is then

$$
\begin{aligned}
\gamma^{\prime(0)} & =R \gamma^{(0)} R^{-1}, \\
\gamma^{\prime(e)} & =R \gamma^{(e)} R^{-1}, \\
\gamma^{\prime(s e)} & =R \gamma^{(s e)} R^{-1}-\left[Z_{Q Q}^{\prime(e, 0)}, \gamma^{\prime(0)}\right]-\left[Z_{Q Q}^{\prime(1,0)}, \gamma^{\prime(e)}\right],
\end{aligned}
$$

in agreement with the results given in ref. [28]. The Wilson coefficients change according to

$$
C^{\prime}=\left[1+\frac{\alpha_{s}}{4 \pi} Z_{Q Q}^{\prime(1,0)}+\frac{\alpha}{4 \pi} Z_{Q Q}^{\prime(e, 0)}\right]^{T}\left(R^{-1}\right)^{T} C
$$

In particular, we find

$$
\begin{aligned}
C^{\prime(e)} & =C^{(e)}, \\
C^{\prime(s e)} & =C^{(s e)}+C^{(0)} Z_{Q Q}^{\prime(e, 0)}+C^{(e)} Z_{Q Q}^{\prime(1,0)} .
\end{aligned}
$$

The matrix elements change with the inverse transformation, thus ensuring the scheme dependence cancels in the amplitude [10]. Hence, we only need to show that the RG evolution does not upend this cancellation. We will consider only the effects of order $\alpha / \alpha_{s}$ and $\alpha$. (The QCD case has been discussed extensively in the literature, see e.g. refs. [3335]. The proof of scheme dependence including QED in ref. [28] fails in our case as the anomalous dimension is itself scheme independent.) 
We expand the hadronic matrix element in powers of couplings as ${ }^{4}$

$$
\langle Q\rangle(\mu)=\left(1+\frac{\alpha_{s}(\mu)}{4 \pi} r^{(1)}+\frac{\alpha}{4 \pi} r^{(s e)}\right)\langle Q\rangle^{(0)} .
$$

Recalling the perturbative expansion of the RG evolution matrix, eq. (4.3), and the expansion in powers of couplings of the low-energy Wilson coefficient, eq. (4.4), the terms at order $\alpha / \alpha_{s}$ and $\alpha$ in the amplitude are then (we drop obvious function arguments)

$$
\begin{aligned}
\langle C U Q\rangle^{(e)}= & \frac{\alpha(\mu)}{\alpha_{s}(\mu)}\left(C^{(0)} U^{(e)}+\eta^{-1} C^{(e)} U^{(0)}\right)\langle Q\rangle^{(0)} \\
\langle C U Q\rangle^{(s e)}=\alpha(\mu)\left(U^{(e)}\left[\eta C^{(1)}+C^{(0)} r^{(1)}\right]+\eta^{-1} C^{(e)}\left[U^{(1)}+U^{(0)} r^{(1)}\right]\right. & \\
& \left.+C^{(0)} U^{(s e)}+C^{(s e)} U^{(0)}+C^{(0)} U^{(0)} r^{(s e)}\right)\langle Q\rangle^{(0)}
\end{aligned}
$$

It is apparent that the order $\alpha / \alpha_{s}$ contribution is scheme independent, as it involves only scheme-independent quantities. In the order $\alpha$ contribution, the QCD scheme dependence cancels within the square brackets in the first line, respectively, as shown in ref. [34]. In the second line, note that the first term is scheme independent. The renormalized matrix element $r^{(s e)}$ is given by ${ }^{5}$

$$
r^{(s e)}=r^{(s e), \text { bare }}+Z^{(e)} .
$$

According to the discussion at the beginning of this section, under a change of renormalization scheme with nonzero coefficient $U$ in eq. (C.1), this contribution to the matrix element transforms as $r^{(s e)} \rightarrow r^{(s e)}-Z^{\prime(e)}$. This finishes the proof of the scheme independence of the matrix element.

Note that for the cancellation to work, we need to fix the electromagnetic coupling in the effective theory to its value at $\mu=M_{Z}$, i.e. $\alpha=\alpha\left(M_{Z}\right)$. This is consistent with the fixed-order perturbative expansion in $\alpha$.

\section{The full two-loop result}

$$
\begin{aligned}
C_{S 2}^{t t,(s e)}= & {\left[128 y-896 y z-384 x y z+288 x z^{2}+2752 y z^{2}+1504 x y z^{2}+448 x^{2} y z^{2}\right.} \\
& -1080 x^{2} z^{3}-7536 x y z^{3}+872 x^{2} y z^{3}-256 x^{3} y z^{3}-576 y^{2} z^{3}+144 x z^{4} \\
& -864 x^{3} z^{4}+608 y z^{4}-1240 x y z^{4}+1564 x^{2} y z^{4}-1664 x^{3} y z^{4}+64 x^{4} y z^{4} \\
& +2016 x y^{2} z^{4}+288 y^{3} z^{4}-540 x^{2} z^{5}+6408 x^{4} z^{5}-1632 x y z^{5}+4740 x^{2} y z^{5} \\
& -10694 x^{3} y z^{5}+184 x^{4} y z^{5}-3438 x^{2} y^{2} z^{5}-720 x y^{3} z^{5}+432 x^{3} z^{6} \\
& -5184 x^{5} z^{6}+2117 x^{2} y z^{6}-1668 x^{3} y z^{6}-4050 x^{4} y z^{6}+1872 x^{3} y^{2} z^{6}
\end{aligned}
$$

\footnotetext{
${ }^{4}$ Of course, in reality this matrix element needs to be computed using nonperturbative methods such as lattice QCD. One can think of eq. (C.10) as the perturbative conversion of the lattice result to the $\overline{\mathrm{MS}}$ scheme.

${ }^{5}$ We assume that parameter and field renormalizations have been performed in the usual way.
} 


$$
\begin{aligned}
& +765 x^{2} y^{3} z^{6}-36 x^{4} z^{7}+432 x^{6} z^{7}-373 x^{3} y z^{7}+364 x^{4} y z^{7} \\
& \left.+1080 x^{5} y z^{7}-198 x^{4} y^{2} z^{7}-225 x^{3} y^{3} z^{7}\right] /\left[288 y(z-1) z(x z-1)^{3}\right] \\
& +\pi^{2}\left[-16 x+128 x z+16 x^{2} z-404 x z^{2}-24 x^{2} z^{2}+8 x^{3} z^{2}+472 x z^{3}-156 x^{2} z^{3}\right. \\
& -8 x^{3} z^{3}+32 z^{4}-32 x z^{4}+148 x^{2} z^{4}-134 x^{3} z^{4}+48 x^{4} z^{4}+8 x^{5} z^{4}-16 z^{5} \\
& +64 x z^{5}-24 x^{2} z^{5}+44 x^{3} z^{5}-24 x^{4} z^{5}+8 x^{5} z^{5}+2 z^{6}-14 x z^{6}+2 x^{2} z^{6} \\
& \left.-96 x^{4} z^{6}+2 x^{5} z^{6}-18 x^{2} y^{2} z^{6}-6 x^{2} z^{7}-108 x^{5} z^{7}-x^{2} z^{8}\right] /\left[216 x^{2}(z-1) z^{3}\right] \\
& +\Phi\left(\frac{1}{4 x}\right)\left[-256 z+2816 x z-10624 x^{2} z+15232 x^{3} z-8192 x^{4} z-2048 x^{5} z\right. \\
& +128 z^{2}-512 x z^{2}-3584 x^{2} z^{2}+21888 x^{3} z^{2}-27520 x^{4} z^{2}+11264 x^{5} z^{2} \\
& +10240 x^{6} z^{2}-16 z^{3}-32 x z^{3}+1304 x^{2} z^{3}-4752 x^{3} z^{3}+2928 x^{4} z^{3} \\
& -12704 x^{5} z^{3}+1024 x^{6} z^{3}-14848 x^{7} z^{3}+32 x z^{4}-1056 x^{2} z^{4}+5936 x^{3} z^{4} \\
& -4640 x^{4} z^{4}-27952 x^{5} z^{4}+72256 x^{6} z^{4}-3840 x^{7} z^{4}+7168 x^{8} z^{4} \\
& -8 x^{2} z^{5}+2304 x^{3} z^{5}-19252 x^{4} z^{5}+50320 x^{5} z^{5}-35164 x^{6} z^{5} \\
& -40352 x^{7} z^{5}+19456 x^{8} z^{5}-512 x^{9} z^{5}-1532 x^{4} z^{6}+11756 x^{5} z^{6} \\
& -23860 x^{6} z^{6}+4184 x^{7} z^{6}+13696 x^{8} z^{6}-2816 x^{9} z^{6}+41 x^{4} z^{7} \\
& \left.-198 x^{5} z^{7}-250 x^{6} z^{7}+1612 x^{7} z^{7}-252 x^{8} z^{7}-1280 x^{9} z^{7}\right] \\
& /\left[576 x^{2}(4 x-1)(z-1)(x z-1)^{4}\right] \\
& +\Phi\left(\frac{y}{4 x}\right)\left[-176 x^{2} z^{3}+256 x y z^{3}-56 y^{2} z^{3}+656 x^{3} z^{4}-1088 x^{2} y z^{4}+304 x y^{2} z^{4}\right. \\
& -16 y^{3} z^{4}-1164 x^{4} z^{5}+1740 x^{3} y z^{5}-564 x^{2} y^{2} z^{5}+48 x y^{3} z^{5}+728 x^{5} z^{6} \\
& -948 x^{4} y z^{6}+324 x^{3} y^{2} z^{6}-32 x^{2} y^{3} z^{6}-44 x^{6} z^{7}+112 x^{5} y z^{7} \\
& \left.-66 x^{4} y^{2} z^{7}+14 x^{3} y^{3} z^{7}-x^{2} y^{4} z^{7}\right] /\left[64(z-1)(x z-1)^{4}\right] \\
& +\Phi\left(\frac{z}{4}\right)\left[256 x^{2} z+7872 x^{2} z^{2}-512 x^{3} z^{2}-23072 x^{2} z^{3}-17216 x^{3} z^{3}+256 x^{4} z^{3}\right. \\
& +5664 x^{2} z^{4}-1680 x^{3} z^{4}+2048 x^{4} z^{4}+5688 x^{2} z^{5}-6240 x^{3} z^{5} \\
& -2768 x^{4} z^{5}-1222 x^{2} z^{6}-1636 x^{3} z^{6}+2032 x^{4} z^{6}-73 x^{2} z^{7} \\
& \left.+900 x^{3} z^{7}+322 x^{4} z^{7}+32 x^{3} z^{8}-208 x^{4} z^{8}-8 x^{4} z^{9}\right] \\
& /\left[576(z-1)(x z-1)^{4}\right] \\
& +\Phi\left(\frac{y z}{4}\right)\left[144 x^{2} z^{3}+48 x^{3} z^{4}-224 x^{2} y z^{4}-192 x^{4} z^{5}+336 x^{3} y z^{5}+132 x^{2} y^{2} z^{5}\right. \\
& -88 x^{4} y z^{6}-228 x^{3} y^{2} z^{6}-18 x^{2} y^{3} z^{6}+98 x^{4} y^{2} z^{7}+36 x^{3} y^{3} z^{7} \\
& \left.-x^{2} y^{4} z^{7}-16 x^{4} y^{3} z^{8}\right] /\left[64(z-1)(x z-1)^{4}\right]
\end{aligned}
$$




$$
\begin{aligned}
& +\Phi\left(\frac{1}{x z}, \frac{1}{x}\right)\left[-64+576 z+384 x z-2128 z^{2}-2848 x z^{2}-928 x^{2} z^{2}+3824 z^{3}\right. \\
& +8704 x z^{3}+5664 x^{2} z^{3}+1120 x^{3} z^{3}-3312 z^{4}-12752 x z^{4} \\
& -14056 x^{2} z^{4}-5816 x^{3} z^{4}-672 x^{4} z^{4}+992 z^{5}+9472 x z^{5} \\
& +14904 x^{2} z^{5}+11672 x^{3} z^{5}+3544 x^{4} z^{5}+224 x^{5} z^{5}+272 z^{6} \\
& -3032 x z^{6}-6904 x^{2} z^{6}-9866 x^{3} z^{6}-6800 x^{4} z^{6}-1840 x^{5} z^{6} \\
& -224 x^{6} z^{6}-144 z^{7}-424 x z^{7}+2232 x^{2} z^{7}+4678 x^{3} z^{7} \\
& +6914 x^{4} z^{7}+4536 x^{5} z^{7}+1104 x^{6} z^{7}+288 x^{7} z^{7}-16 z^{8} \\
& +456 x z^{8}-592 x^{2} z^{8}-607 x^{3} z^{8}+6444 x^{4} z^{8}+368 x^{5} z^{8} \\
& -2664 x^{6} z^{8}-408 x^{7} z^{8}-160 x^{8} z^{8}+40 x z^{9}-304 x^{2} z^{9} \\
& -1787 x^{3} z^{9}+2891 x^{4} z^{9}+2268 x^{5} z^{9}-2720 x^{6} z^{9} \\
& +1000 x^{7} z^{9}-8 x^{8} z^{9}+32 x^{9} z^{9}-16 x^{2} z^{10}+565 x^{3} z^{10} \\
& -1766 x^{4} z^{10}+2963 x^{5} z^{10}-2524 x^{6} z^{10}+1018 x^{7} z^{10} \\
& -272 x^{8} z^{10}+32 x^{9} z^{10}+41 x^{3} z^{11}-403 x^{4} z^{11} \\
& \left.+1093 x^{5} z^{11}-1279 x^{6} z^{11}+694 x^{7} z^{11}-154 x^{8} z^{11}+8 x^{9} z^{11}\right] \\
& /\left[288 x^{2}(z-1) z^{4}(x z-1)^{4}\right] \\
& +\Phi\left(\frac{1}{x z}, \frac{y}{x}\right)\left[-16+104 x z+64 y z-296 x^{2} z^{2}-296 x y z^{2}-96 y^{2} z^{2}\right. \\
& +476 x^{3} z^{3}+544 x^{2} y z^{3}+344 x y^{2} z^{3}+64 y^{3} z^{3}-436 x^{4} z^{4} \\
& -568 x^{3} y z^{4}-416 x^{2} y^{2} z^{4}-216 x y^{3} z^{4}-16 y^{4} z^{4}+160 x^{5} z^{5} \\
& +326 x^{4} y z^{5}+271 x^{3} y^{2} z^{5}+192 x^{2} y^{3} z^{5}+64 x y^{4} z^{5}+80 x^{6} z^{6} \\
& -106 x^{5} y z^{6}-185 x^{4} y^{2} z^{6}+19 x^{3} y^{3} z^{6}-88 x^{2} y^{4} z^{6}-100 x^{7} z^{7} \\
& +114 x^{6} y z^{7}-31 x^{5} y^{2} z^{7}-24 x^{4} y^{3} z^{7}+41 x^{3} y^{4} z^{7}+28 x^{8} z^{8} \\
& \left.-78 x^{7} y z^{8}+65 x^{6} y^{2} z^{8}-7 x^{5} y^{3} z^{8}-9 x^{4} y^{4} z^{8}+x^{3} y^{5} z^{8}\right] \\
& /\left[32 x^{2}(z-1) z(x z-1)^{4}\right] \\
& +\mathrm{Li}_{2}\left(1-\frac{1}{z}\right)\left[16-112 z-48 x z+292 z^{2}+232 x z^{2}+40 x^{2} z^{2}-260 z^{3}\right. \\
& -420 x z^{3}-128 x^{2} z^{3}+16 z^{4}+236 x z^{4}+114 x^{2} z^{4}-40 x^{3} z^{4} \\
& +44 z^{5}-64 x z^{5}+186 x^{2} z^{5}+72 x^{3} z^{5}+4 z^{6}-42 x z^{6}+8 x^{2} z^{6} \\
& \left.-155 x^{3} z^{6}-2 x z^{7}-54 x^{2} z^{7}+31 x^{3} z^{7}-4 x^{2} z^{8}+36 x^{3} z^{8}+2 x^{3} z^{9}\right] \\
& /\left[36 x z^{3}(x z-1)^{2}\right]
\end{aligned}
$$




$$
\begin{aligned}
& +\mathrm{Li}_{2}(1-y z)\left[4-14 x z-12 y z+20 x^{2} z^{2}+24 x y z^{2}+12 y^{2} z^{2}-13 x^{3} z^{3}\right. \\
& -12 x^{2} y z^{3}-18 x y^{2} z^{3}-4 y^{3} z^{3}+6 x^{3} y z^{4}-6 x^{2} y^{2} z^{4}+8 x y^{3} z^{4} \\
& \left.+9 x^{3} y^{2} z^{5}-2 x^{2} y^{3} z^{5}-2 x^{3} y^{3} z^{6}\right] /\left[4 x(z-1)(x z-1)^{2}\right] \\
& +\mathrm{Li}_{2}(1-x z)\left[-32+256 z+128 x z-808 z^{2}-816 x z^{2}-176 x^{2} z^{2}+944 z^{3}\right. \\
& +2112 x z^{3}+896 x^{2} z^{3}+80 x^{3} z^{3}-2504 x z^{4}-1788 x^{2} z^{4} \\
& -396 x^{3} z^{4}+1968 x^{2} z^{5}+616 x^{3} z^{5}+164 x^{4} z^{5}+32 x^{5} z^{5} \\
& -3 x^{3} z^{6}-644 x^{4} z^{6}-164 x^{5} z^{6}-48 x^{6} z^{6}-375 x^{4} z^{7}+332 x^{5} z^{7} \\
& +44 x^{6} z^{7}+16 x^{7} z^{7}+25 x^{5} z^{8}-112 x^{6} z^{8}+16 x^{7} z^{8}+449 x^{6} z^{9} \\
& \left.+4 x^{7} z^{9}-216 x^{7} z^{10}\right] /\left[72 x(z-1) z^{3}(x z-1)^{3}\right] \\
& +\mathrm{Li}_{2}\left(1-\frac{x}{y}\right)\left[9 x^{2} z^{3}-18 x y z^{3}+9 y^{2} z^{3}-20 x^{3} z^{4}+42 x^{2} y z^{4}-24 x y^{2} z^{4}\right. \\
& \left.+2 y^{3} z^{4}+8 x^{4} z^{5}-18 x^{3} y z^{5}+12 x^{2} y^{2} z^{5}-2 x y^{3} z^{5}\right] \\
& /\left[4(z-1)(x z-1)^{2}\right] \\
& +\mathrm{Li}_{2}(1-x)\left[-64 z+192 x z-160 x^{2} z+16 x^{3} z+16 x^{5} z+32 z^{2}-32 x z^{2}\right. \\
& -72 x^{2} z^{2}-16 x^{3} z^{2}+200 x^{4} z^{2}-96 x^{5} z^{2}-16 x^{6} z^{2}-4 z^{3} \\
& -2 x z^{3}+141 x^{2} z^{3}-256 x^{3} z^{3}+165 x^{4} z^{3}-84 x^{5} z^{3}+32 x^{6} z^{3} \\
& +8 x^{7} z^{3}+4 x z^{4}-98 x^{2} z^{4}+136 x^{3} z^{4}-6 x^{4} z^{4}-16 x^{5} z^{4} \\
& -28 x^{6} z^{4}+8 x^{7} z^{4}+84 x^{3} z^{5}-188 x^{4} z^{5}+126 x^{5} z^{5} \\
& \left.-24 x^{6} z^{5}+2 x^{7} z^{5}\right] /\left[36 x^{2}(z-1)(x z-1)^{2}\right] \\
& +\log ^{2}(x)\left[256 y z-1280 x y z+1408 x^{2} y z-128 x^{3} y z-128 x^{5} y z-128 y z^{2}\right. \\
& -512 x y z^{2}+4096 x^{2} y z^{2}-4352 x^{3} y z^{2}-1216 x^{4} y z^{2}+768 x^{5} y z^{2} \\
& +512 x^{6} y z^{2}+16 y z^{3}+256 x y z^{3}-376 x^{2} y z^{3}-2688 x^{3} y z^{3} \\
& +3776 x^{4} y z^{3}+8160 x^{5} y z^{3}-2560 x^{6} y z^{3}-832 x^{7} y z^{3}+1296 x^{3} y^{2} z^{3} \\
& -504 x^{2} y^{3} z^{3}-48 x y z^{4}+736 x^{2} y z^{4}+72 x^{3} y z^{4}-3904 x^{4} y z^{4} \\
& -7296 x^{5} y z^{4}-12608 x^{6} y z^{4}+3008 x^{7} y z^{4}+704 x^{8} y z^{4}-6480 x^{4} y^{2} z^{4} \\
& +2952 x^{3} y^{3} z^{4}-144 x^{2} y^{4} z^{4}-5184 x^{7} z^{5}+40 x^{2} y z^{5}-3120 x^{3} y z^{5} \\
& +6228 x^{4} y z^{5}+968 x^{5} y z^{5}+13272 x^{6} y z^{5}+6000 x^{7} y z^{5}-1344 x^{8} y z^{5} \\
& -320 x^{9} y z^{5}+13284 x^{5} y^{2} z^{5}-6660 x^{4} y^{3} z^{5}+576 x^{3} y^{4} z^{5} \\
& +10368 x^{8} z^{6}-8 x^{3} y z^{6}+3788 x^{4} y z^{6}-8232 x^{5} y z^{6}+2092 x^{6} y z^{6} \\
& -2760 x^{7} y z^{6}+48 x^{8} y z^{6}+64 x^{9} y z^{6}+64 x^{10} y z^{6}-12528 x^{6} y^{2} z^{6} \\
& +6552 x^{5} y^{3} z^{6}-720 x^{4} y^{4} z^{6}-5184 x^{9} z^{7}-41 x^{4} y z^{7}-1580 x^{5} y z^{7}
\end{aligned}
$$




$$
\begin{aligned}
& +2772 x^{6} y z^{7}+804 x^{7} y z^{7}-5584 x^{8} y z^{7}-272 x^{9} y z^{7}+64 x^{10} y z^{7} \\
& +4824 x^{7} y^{2} z^{7}-2736 x^{6} y^{3} z^{7}+396 x^{5} y^{4} z^{7}-9 x^{4} y^{5} z^{7}+41 x^{5} y z^{8} \\
& +48 x^{6} y z^{8}-208 x^{7} y z^{8}-88 x^{8} y z^{8}+3696 x^{9} y z^{8}+16 x^{10} y z^{8} \\
& \left.-396 x^{8} y^{2} z^{8}+396 x^{7} y^{3} z^{8}-108 x^{6} y^{4} z^{8}+9 x^{5} y^{5} z^{8}-864 x^{10} y z^{9}\right] \\
& /\left[576 x^{2} y(z-1)(x z-1)^{5}\right] \\
& +\log ^{2}(y)\left[9 x^{2} z^{3}-18 x y z^{3}+7 y^{2} z^{3}-20 x^{3} z^{4}+42 x^{2} y z^{4}-20 x y^{2} z^{4}+2 y^{3} z^{4}\right. \\
& \left.+8 x^{4} z^{5}-18 x^{3} y z^{5}+10 x^{2} y^{2} z^{5}-2 x y^{3} z^{5}\right] /\left[8(z-1)(x z-1)^{2}\right] \\
& +\log (z)\left[128 y-832 y z-448 x y z+1776 y z^{2}+2976 x y z^{2}+512 x^{2} y z^{2}-192 y z^{3}\right. \\
& -6256 x y z^{3}-4784 x^{2} y z^{3}-160 x^{3} y z^{3}-144 y^{2} z^{3}+216 x z^{4} \\
& -648 x^{3} z^{4}-16 y z^{4}+232 x y z^{4}+10336 x^{2} y z^{4}+4032 x^{3} y z^{4} \\
& -64 x^{4} y z^{4}+684 x y^{2} z^{4}-1026 x^{2} z^{5}+3888 x^{4} z^{5}+40 x y z^{5} \\
& +268 x^{2} y z^{5}-5374 x^{3} y z^{5}-1576 x^{4} y z^{5}+32 x^{5} y z^{5}-1134 x^{2} y^{2} z^{5} \\
& +1566 x^{3} z^{6}-7128 x^{5} z^{6}-491 x^{2} y z^{6}-1668 x^{3} y z^{6}+9007 x^{4} y z^{6} \\
& +184 x^{5} y z^{6}+1377 x^{3} y^{2} z^{6}-171 x^{2} y^{3} z^{6}-810 x^{4} z^{7}+4104 x^{6} z^{7} \\
& +203 x^{3} y z^{7}+340 x^{4} y z^{7}-1938 x^{5} y z^{7}-648 x^{4} y^{2} z^{7}+189 x^{3} y^{3} z^{7} \\
& +54 x^{5} z^{8}-216 x^{7} z^{8}-96 x^{4} y z^{8}-78 x^{5} y z^{8}+567 x^{6} y z^{8} \\
& \left.+27 x^{5} y^{2} z^{8}-72 x^{4} y^{3} z^{8}\right] /\left[144 y(z-1) z(x z-1)^{4}\right] \\
& +\log ^{2}(z)\left[-288 y+1872 x y z-560 x^{3} y z+864 y^{2} z-5328 x^{2} y z^{2}+1424 x^{3} y z^{2}\right. \\
& +2096 x^{4} y z^{2}+128 x^{5} y z^{2}-4320 x y^{2} z^{2}-864 y^{3} z^{2}+2348 x^{3} y z^{3} \\
& -2672 x^{4} y z^{3}-3168 x^{5} y z^{3}-448 x^{6} y z^{3}+8640 x^{2} y^{2} z^{3}+3888 x y^{3} z^{3} \\
& +288 y^{4} z^{3}+8248 x^{3} y z^{4}-5916 x^{4} y z^{4}+2400 x^{5} y z^{4}+2464 x^{6} y z^{4} \\
& +576 x^{7} y z^{4}-9864 x^{3} y^{2} z^{4}-6048 x^{2} y^{3} z^{4}-1440 x y^{4} z^{4}+1296 x^{4} z^{5} \\
& -5184 x^{6} z^{5}+2806 x^{3} y z^{5}-16040 x^{4} y z^{5}+20288 x^{5} y z^{5}-2928 x^{6} y z^{5} \\
& -944 x^{7} y z^{5}-320 x^{8} y z^{5}+7920 x^{4} y^{2} z^{5}+3618 x^{3} y^{3} z^{5}+2736 x^{2} y^{4} z^{5} \\
& -2592 x^{5} z^{6}+10368 x^{7} z^{6}-1368 x^{3} y z^{6}-2450 x^{4} y z^{6}+9708 x^{5} y z^{6} \\
& -11280 x^{6} y z^{6}+2240 x^{7} y z^{6}+48 x^{8} y z^{6}+64 x^{9} y z^{6}-4212 x^{5} y^{2} z^{6} \\
& -270 x^{4} y^{3} z^{6}-2484 x^{3} y^{4} z^{6}+1296 x^{6} z^{7}-5184 x^{8} z^{7}-73 x^{3} y z^{7} \\
& +2332 x^{4} y z^{7}-498 x^{5} y z^{7}-1916 x^{6} y z^{7}-3060 x^{7} y z^{7}-480 x^{8} y z^{7} \\
& +64 x^{9} y z^{7}+972 x^{6} y^{2} z^{7}-378 x^{5} y^{3} z^{7}+1080 x^{4} y^{4} z^{7}-9 x^{3} y^{5} z^{7} \\
& +105 x^{4} y z^{8}-1188 x^{5} y z^{8}+142 x^{6} y z^{8}+3524 x^{8} y z^{8}+16 x^{9} y z^{8}
\end{aligned}
$$




$$
\begin{aligned}
& +54 x^{6} y^{3} z^{8}-180 x^{5} y^{4} z^{8}+9 x^{4} y^{5} z^{8}-40 x^{5} y z^{9}+224 x^{6} y z^{9} \\
& \left.-864 x^{9} y z^{9}+8 x^{6} y z^{10}\right] /\left[576 x y(z-1)(x z-1)^{5}\right] \\
& +\log \left(\frac{\mu}{M_{W}}\right)\left\{\left[432 x z-1408 x y z-1620 x^{2} z^{2}+1072 x y z^{2}+5280 x^{2} y z^{2}\right.\right. \\
& +216 x z^{3}+432 x^{3} z^{3}-312 x y z^{3}-3732 x^{2} y z^{3}-4224 x^{3} y z^{3} \\
& +108 x y^{2} z^{3}-810 x^{2} z^{4}+3132 x^{4} z^{4}+1323 x^{2} y z^{4}-312 x^{3} y z^{4} \\
& +352 x^{4} y z^{4}-405 x^{2} y^{2} z^{4}+648 x^{3} z^{5}-2592 x^{5} z^{5}-171 x^{3} y z^{5} \\
& +137 x^{4} y z^{5}+324 x^{3} y^{2} z^{5}-54 x^{4} z^{6}+216 x^{6} z^{6}+78 x^{4} y z^{6} \\
& \left.-135 x^{5} y z^{6}-27 x^{4} y^{2} z^{6}\right] /\left[72 y(z-1)(x z-1)^{3}\right] \\
& +\left[108 x^{3} z^{3}-352 x^{3} y z^{3}-108 x^{4} z^{4}+436 x^{3} y z^{4}+352 x^{4} y z^{4}\right. \\
& +54 x^{3} z^{5}-216 x^{5} z^{5}-180 x^{3} y z^{5}+113 x^{4} y z^{5}+27 x^{3} y^{2} z^{5} \\
& \left.-54 x^{4} z^{6}+216 x^{6} z^{6}+27 x^{4} y z^{6}-54 x^{5} y z^{6}-27 x^{4} y^{2} z^{6}\right] \\
& \left.\times \log (x z) /\left[12 y(z-1)(x z-1)^{4}\right]\right\} \\
& +\log (y)\left[-16 y z^{2}+40 x y z^{3}+16 y^{2} z^{3}-30 x^{2} y z^{4}-40 x y^{2} z^{4}+7 x^{3} y z^{5}\right. \\
& \left.+43 x^{2} y^{2} z^{5}-x^{4} y z^{6}-13 x^{3} y^{2} z^{6}\right] /\left[16(z-1)(x z-1)^{3}\right] \\
& +\left[16-88 x z-48 y z+208 x^{2} z^{2}+192 x y z^{2}+48 y^{2} z^{2}-268 x^{3} z^{3}\right. \\
& -288 x^{2} y z^{3}-168 x y^{2} z^{3}-16 y^{3} z^{3}+168 x^{4} z^{4}+284 x^{3} y z^{4} \\
& +168 x^{2} y^{2} z^{4}+64 x y^{3} z^{4}+8 x^{5} z^{5}-210 x^{4} y z^{5}-39 x^{3} y^{2} z^{5} \\
& -88 x^{2} y^{3} z^{5}-72 x^{6} z^{6}+120 x^{5} y z^{6}-48 x^{4} y^{2} z^{6}+58 x^{3} y^{3} z^{6} \\
& \left.+28 x^{7} z^{7}-50 x^{6} y z^{7}+21 x^{5} y^{2} z^{7}-12 x^{4} y^{3} z^{7}\right] \\
& \times \log (z) /\left[32 x(z-1)(x z-1)^{4}\right] \\
& +\log (x)\left\{\left[-64 y+256 x y+448 y z-1600 x y z-768 x^{2} y z-1248 y z^{2}+2528 x y z^{2}\right.\right. \\
& +6688 x^{2} y z^{2}+384 x^{3} y z^{2}-64 y z^{3}+5360 x y z^{3}-10944 x^{2} y z^{3} \\
& -13504 x^{3} y z^{3}+768 x^{4} y z^{3}+288 y^{2} z^{3}-1152 x y^{2} z^{3}+648 x^{3} z^{4} \\
& -2592 x^{4} z^{4}-296 y z^{4}+1496 x y z^{4}-11792 x^{2} y z^{4}+19904 x^{3} y z^{4} \\
& +14048 x^{4} y z^{4}-896 x^{5} y z^{4}-1188 x y^{2} z^{4}+4752 x^{2} y^{2} z^{4}-144 y^{3} z^{4} \\
& +576 x y^{3} z^{4}-3888 x^{4} z^{5}+15552 x^{5} z^{5}+1104 x y z^{5}-4900 x^{2} y z^{5} \\
& +11174 x^{3} y z^{5}-7328 x^{4} y z^{5}-6432 x^{5} y z^{5}+256 x^{6} y z^{5}+1764 x^{2} y^{2} z^{5} \\
& -7056 x^{3} y^{2} z^{5}+504 x y^{3} z^{5}-2016 x^{2} y^{3} z^{5}-108 x^{3} z^{6}+432 x^{4} z^{6} \\
& +7128 x^{5} z^{6}-28512 x^{6} z^{6}-1432 x^{2} y z^{6}+7409 x^{3} y z^{6}-20447 x^{4} y z^{6}
\end{aligned}
$$




$$
\begin{aligned}
& +33124 x^{5} y z^{6}+864 x^{6} y z^{6}-1710 x^{3} y^{2} z^{6}+6840 x^{4} y^{2} z^{6}-576 x^{2} y^{3} z^{6} \\
& +2304 x^{3} y^{3} z^{6}+108 x^{4} z^{7}-432 x^{5} z^{7}-4104 x^{6} z^{7}+16416 x^{7} z^{7} \\
& +1099 x^{3} y z^{7}-4722 x^{4} y z^{7}+5226 x^{5} y z^{7}-7528 x^{6} y z^{7}+720 x^{4} y^{2} z^{7} \\
& -2880 x^{5} y^{2} z^{7}+315 x^{3} y^{3} z^{7}-1260 x^{4} y^{3} z^{7}+216 x^{7} z^{8}-864 x^{8} z^{8} \\
& -115 x^{4} y z^{8}+439 x^{5} y z^{8}-783 x^{6} y z^{8}+2268 x^{7} y z^{8}-36 x^{5} y^{2} z^{8} \\
& \left.+144 x^{6} y^{2} z^{8}-45 x^{4} y^{3} z^{8}+180 x^{5} y^{3} z^{8}\right] \\
& /\left[144(4 x-1) y(z-1) z(x z-1)^{4}\right] \\
& +\left[16-88 x z-48 y z+208 x^{2} z^{2}+192 x y z^{2}+48 y^{2} z^{2}-340 x^{3} z^{3}\right. \\
& -144 x^{2} y z^{3}-224 x y^{2} z^{3}-16 y^{3} z^{3}+472 x^{4} z^{4}-340 x^{3} y z^{4} \\
& +440 x^{2} y^{2} z^{4}+48 x y^{3} z^{4}-448 x^{5} z^{5}+750 x^{4} y z^{5}-495 x^{3} y^{2} z^{5} \\
& -40 x^{2} y^{3} z^{5}+216 x^{6} z^{6}-504 x^{5} y z^{6}+272 x^{4} y^{2} z^{6}+10 x^{3} y^{3} z^{6} \\
& \left.-36 x^{7} z^{7}+94 x^{6} y z^{7}-59 x^{5} y^{2} z^{7}+4 x^{4} y^{3} z^{7}\right] \\
& \times \log (y) /\left[32 x(z-1)(x z-1)^{4}\right] \\
& +\left[-64 y+512 y z+384 x y z-1616 y z^{2}-2656 x y z^{2}-928 x^{2} y z^{2}\right. \\
& +2064 y z^{3}+7456 x y z^{3}+5568 x^{2} y z^{3}+1120 x^{3} y z^{3}-1104 y z^{4} \\
& -8312 x y z^{4}-13640 x^{2} y z^{4}-6008 x^{3} y z^{4}-672 x^{4} y z^{4}+432 y^{2} z^{4} \\
& -112 y z^{5}+4512 x y z^{5}+11544 x^{2} y z^{5}+12608 x^{3} y z^{5}+3704 x^{4} y z^{5} \\
& +224 x^{5} y z^{5}-2160 x y^{2} z^{5}-432 y^{3} z^{5}+160 y z^{6}+248 x y z^{6} \\
& -6200 x^{2} y z^{6}-6630 x^{3} y z^{6}-3968 x^{4} y z^{6}-1776 x^{5} y z^{6}-224 x^{6} y z^{6} \\
& +4320 x^{2} y^{2} z^{6}+1944 x y^{3} z^{6}+144 y^{4} z^{6}+16 y z^{7}-640 x y z^{7} \\
& +176 x^{2} y z^{7}+5732 x^{3} y z^{7}-10218 x^{4} y z^{7}-2248 x^{5} y z^{7}+1072 x^{6} y z^{7} \\
& +288 x^{7} y z^{7}-4716 x^{3} y^{2} z^{7}-3024 x^{2} y^{3} z^{7}-720 x y^{4} z^{7}+648 x^{4} z^{8} \\
& -5184 x^{6} z^{8}-56 x y z^{8}+760 x^{2} y z^{8}+1197 x^{3} y z^{8}-6256 x^{4} y z^{8} \\
& +25032 x^{5} y z^{8}+520 x^{6} y z^{8}-472 x^{7} y z^{8}-160 x^{8} y z^{8}+3474 x^{4} y^{2} z^{8} \\
& +1755 x^{3} y^{3} z^{8}+1368 x^{2} y^{4} z^{8}-1296 x^{5} z^{9}+10368 x^{7} z^{9}+56 x^{2} y z^{9} \\
& -870 x^{3} y z^{9}-53 x^{4} y z^{9}+2044 x^{5} y z^{9}-11776 x^{6} y z^{9}+1120 x^{7} y z^{9} \\
& +24 x^{8} y z^{9}+32 x^{9} y z^{9}-1242 x^{5} y^{2} z^{9}-351 x^{4} y^{3} z^{9}-1170 x^{3} y^{4} z^{9} \\
& +648 x^{6} z^{10}-5184 x^{8} z^{10}-57 x^{3} y z^{10}+952 x^{4} y z^{10}-2187 x^{5} y z^{10} \\
& +1820 x^{6} y z^{10}-2962 x^{7} y z^{10}-240 x^{8} y z^{10}+32 x^{9} y z^{10} \\
& -558 x^{6} y^{2} z^{10}+243 x^{5} y^{3} z^{10}+450 x^{4} y^{4} z^{10}-9 x^{3} y^{5} z^{10}
\end{aligned}
$$




$$
\begin{aligned}
& +41 x^{4} y z^{11}-362 x^{5} y z^{11}+731 x^{6} y z^{11}-548 x^{7} y z^{11} \\
& +3418 x^{8} y z^{11}+8 x^{9} y z^{11}+450 x^{7} y^{2} z^{11}-135 x^{6} y^{3} z^{11} \\
& \left.-72 x^{5} y^{4} z^{11}+9 x^{4} y^{5} z^{11}-864 x^{9} y z^{12}\right] \\
& \left.\times \log (z) /\left[288 x y(z-1) z^{3}(x z-1)^{5}\right]\right\} .
\end{aligned}
$$

Here, $x \equiv m_{t}^{2} / M_{Z}^{2}, y \equiv M_{h}^{2} / M_{Z}^{2}$, and $z \equiv M_{Z}^{2} / M_{W}^{2}$. The functions $\Phi$ are defined in ref. [17].

Open Access. This article is distributed under the terms of the Creative Commons Attribution License (CC-BY 4.0), which permits any use, distribution and reproduction in any medium, provided the original author(s) and source are credited.

\section{References}

[1] K. Anikeev et al., B physics at the Tevatron: Run II and beyond, in proceedings of the Workshop on B Physics at the Tevatron: Run II and Beyond, Batavia, IL, U.S.A., 23-25 September 1999, hep-ph/0201071 [INSPIRE].

[2] Particle Data collaboration, Review of Particle Physics, Prog. Theor. Exp. Phys. 2020 (2020) 083C01 [INSPIRE].

[3] Flavour Lattice Averaging Group, FLAG Review 2019: Flavour Lattice Averaging Group (FLAG), Eur. Phys. J. C 80 (2020) 113 [arXiv: 1902.08191] [inSPIRE].

[4] A.J. Buras, D. Guadagnoli and G. Isidori, On $\epsilon_{K}$ Beyond Lowest Order in the Operator Product Expansion, Phys. Lett. B 688 (2010) 309 [arXiv:1002.3612] [INSPIRE].

[5] T. Inami and C.S. Lim, Effects of Superheavy Quarks and Leptons in Low-Energy Weak Processes $K_{L} \rightarrow \mu \bar{\mu}, K^{+} \rightarrow \pi^{+} \nu \bar{\nu}$ and $K^{0} \leftrightarrow \bar{K}^{0}$, Prog. Theor. Phys. 65 (1981) 297 [Erratum ibid. 65 (1981) 1772] [INSPIRE].

[6] G. Buchalla, A.J. Buras and M.E. Lautenbacher, Weak decays beyond leading logarithms, Rev. Mod. Phys. 68 (1996) 1125 [hep-ph/9512380] [INSPIRE].

[7] J. Brod, M. Gorbahn and E. Stamou, Standard-Model Prediction of $\epsilon_{K}$ with Manifest Quark-Mixing Unitarity, Phys. Rev. Lett. 125 (2020) 171803 [arXiv:1911.06822] [INSPIRE].

[8] A.J. Buras, M. Jamin and P.H. Weisz, Leading and Next-to-leading QCD Corrections to $\epsilon$ Parameter and $B^{0}-\bar{B}^{0}$ Mixing in the Presence of a Heavy Top Quark, Nucl. Phys. B 347 (1990) 491 [INSPIRE].

[9] S. Herrlich and U. Nierste, Enhancement of the $K_{L}-K_{S}$ mass difference by short distance QCD corrections beyond leading logarithms, Nucl. Phys. B 419 (1994) 292 [hep-ph/9310311] [INSPIRE].

[10] J. Brod and M. Gorbahn, $\epsilon_{K}$ at Next-to-Next-to-Leading Order: The Charm-Top-Quark Contribution, Phys. Rev. D 82 (2010) 094026 [arXiv: 1007.0684] [InSPIRE].

[11] J. Brod and M. Gorbahn, Next-to-Next-to-Leading-Order Charm-Quark Contribution to the $C P$ Violation Parameter $\epsilon_{K}$ and $\Delta M_{K}$, Phys. Rev. Lett. 108 (2012) 121801 [arXiv: 1108.2036] [INSPIRE].

[12] J. Brod, M. Gorbahn, E. Stamou and H. Yu, work in progress. 
[13] P. Gambino, A. Kwiatkowski and N. Pott, Electroweak effects in the ${ }^{B} 0-\bar{B}^{0}$ mixing, Nucl. Phys. B 544 (1999) 532 [hep-ph/9810400] [INSPIRE].

[14] C. Bobeth, M. Gorbahn and E. Stamou, Electroweak Corrections to $B_{s, d} \rightarrow \ell^{+} \ell^{-}$, Phys. Rev. D 89 (2014) 034023 [arXiv: 1311.1348] [INSPIRE].

[15] A.J. Buras, P.H. Chankowski, J. Rosiek and L. Slawianowska, $\Delta M_{d, s}, B^{0} d, s \rightarrow \mu^{+} \mu^{-}$and $B \rightarrow X_{s} \gamma$ in supersymmetry at large $\tan \beta$, Nucl. Phys. B 659 (2003) 3 [hep-ph/0210145] [INSPIRE].

[16] J.A.M. Vermaseren, New features of FORM, math-ph/0010025 [INSPIRE].

[17] A.I. Davydychev and J.B. Tausk, Two loop selfenergy diagrams with different masses and the momentum expansion, Nucl. Phys. B 397 (1993) 123 [InSPIRE].

[18] C. Bobeth, M. Misiak and J. Urban, Photonic penguins at two loops and $m_{t}$ dependence of $B R\left[B \rightarrow X_{s} l^{+} l^{-}\right]$, Nucl. Phys. B 574 (2000) 291 [hep-ph/9910220] [INSPIRE].

[19] P. Nogueira, Automatic Feynman graph generation, J. Comput. Phys. 105 (1993) 279 [INSPIRE].

[20] A. Sirlin, Large $m_{W}, m_{Z}$ Behavior of the $O(\alpha)$ Corrections to Semileptonic Processes Mediated by W, Nucl. Phys. B 196 (1982) 83 [InSPIRE].

[21] T. van Ritbergen and R.G. Stuart, On the precise determination of the Fermi coupling constant from the muon lifetime, Nucl. Phys. B 564 (2000) 343 [hep-ph/9904240] [InSPIRE].

[22] K.G. Chetyrkin, J.H. Kühn and M. Steinhauser, RunDec: A Mathematica package for running and decoupling of the strong coupling and quark masses, Comput. Phys. Commun. 133 (2000) 43 [hep-ph/0004189] [INSPIRE].

[23] F. Jegerlehner, M.Y. Kalmykov and O. Veretin, MS versus pole masses of gauge bosons: Electroweak bosonic two loop corrections, Nucl. Phys. B 641 (2002) 285 [hep-ph/0105304] [INSPIRE].

[24] F. Jegerlehner, M.Y. Kalmykov and O. Veretin, MS-bar versus pole masses of gauge bosons. 2. Two loop electroweak fermion corrections, Nucl. Phys. B 658 (2003) 49 [hep-ph/0212319] [INSPIRE].

[25] H. Arason et al., Renormalization group study of the standard model and its extensions. 1. The Standard model, Phys. Rev. D 46 (1992) 3945 [InSPIRE].

[26] J. Brod and Z. Polonsky, Two-loop $\beta$-function for Complex Scalar Electroweak Multiplets, JHEP 09 (2020) 158 [arXiv: 2007.13755] [InSPIRE].

[27] M. Awramik, M. Czakon, A. Freitas and G. Weiglein, Precise prediction for the $W$ boson mass in the standard model, Phys. Rev. D 69 (2004) 053006 [hep-ph/0311148] [INSPIRE].

[28] A.J. Buras, M. Jamin and M.E. Lautenbacher, The Anatomy of $\epsilon^{\prime} / \epsilon$ beyond leading logarithms with improved hadronic matrix elements, Nucl. Phys. B 408 (1993) 209 [hep-ph/9303284] [INSPIRE].

[29] K.G. Chetyrkin, M. Misiak and M. Münz, $\beta$-functions and anomalous dimensions up to three loops, Nucl. Phys. B 518 (1998) 473 [hep-ph/9711266] [INSPIRE].

[30] J. Brod, S. Kvedaraite, Z. Polonsky and A. Youssef, work in progress.

[31] D. Binosi and L. Theussl, JaxoDraw: A Graphical user interface for drawing Feynman diagrams, Comput. Phys. Commun. 161 (2004) 76 [hep-ph/0309015] [INSPIRE]. 
[32] J.A.M. Vermaseren, Axodraw, Comput. Phys. Commun. 83 (1994) 45 [inSPIRE].

[33] M. Gorbahn and U. Haisch, Effective Hamiltonian for non-leptonic $|\Delta F|=1$ decays at NNLO in QCD, Nucl. Phys. B 713 (2005) 291 [hep-ph/0411071] [InSPIRE].

[34] A.J. Buras, M. Jamin, M.E. Lautenbacher and P.H. Weisz, Effective Hamiltonians for $\Delta S=1$ and $\Delta B=1$ nonleptonic decays beyond the leading logarithmic approximation, Nucl. Phys. B 370 (1992) 69 [Addendum ibid. $\mathbf{3 7 5}$ (1992) 501] [INSPIRE].

[35] S. Herrlich and U. Nierste, Evanescent operators, scheme dependences and double insertions, Nucl. Phys. B 455 (1995) 39 [hep-ph/9412375] [INSPIRE]. 